\title{
A NEW FINITE LOOP SPACE AT THE PRIME TWO
}

\author{
W. G. DWYER AND C. W. WILKERSON
}

From the point of view of homotopy theory a compact Lie group $G$ has the following remarkable combination of properties:

(1) $G$ can be given the structure of a finite CW-complex, and

(2) there is a pointed space $B G$ and a homotopy equivalence from $G$ to the loop space $\Omega B G$.

Of course the space $B G$ in (2) is the ordinary classifying space of $G$. In general, a finite complex $X$ together with a chosen equivalence $X \rightarrow \Omega B X$ for some $B X$ is called a finite loop space. If $p$ is a prime number and the geometric finiteness condition on $X$ is replaced by the requirement that $X$ be $\mathbf{F}_{p}$-complete in the sense of [3] and have finite $\bmod p$ cohomology, then $X$ is called a $p$-adic finite loop space or a finite loop space at the prime $p$. A ( $p$-adic) finite loop space is a strong homotopy-theoretic analogue of a compact Lie group. The study of these spaces is related to many classical questions in topology (for instance, to the problem of determining all spaces with polynomial cohomology rings).

Call a $p$-adic finite loop space $X$ exotic if it is not the $\mathbf{F}_{p}$-completion of $G$ for a compact Lie group $G$. There are many known examples of exotic $p$-adic finite loop spaces at odd primes $p$ [5] and the classification of these spaces is partially understood [8,9]. However, until now there have been no known exotic 2-adic finite loop spaces.

Recall [32] that the ring of rank 4 mod 2 Dickson invariants is the ring of invariants of the natural action of $\mathrm{GL}\left(4, \mathbf{F}_{2}\right)$ on the rank 4 polynomial algebra $H^{*}\left((B Z / 2)^{4}, \mathbf{F}_{2}\right)$; this ring of invariants is a polynomial algebra on classes $c_{8}, c_{12}, c_{14}$, and $c_{15}$ with $S q^{4} c_{8}=c_{12}, S q^{2} c_{12}=c_{14}$, and $S q^{1} c_{14}=c_{15}$. Our main theorem is the following one.

1.1. Theorem. There exists an $\mathbf{F}_{2}$-complete space $B \mathrm{DI}(4)$ such that $H^{*}\left(B \mathrm{DI}(4), \mathbf{F}_{2}\right)$ is isomorphic as an algebra over the Steenrod algebra to the ring of rank 4 mod 2 Dickson invariants.

Let $\mathrm{DI}(4)=\Omega B \mathrm{DI}(4)$. Standard methods show that $H^{*}\left(\mathrm{DI}(4), \mathbf{F}_{2}\right)$ is multiplicatively generated by elements $x_{7}, y_{11}$, and $z_{13}$, with $S q^{4} x=y$, $S q^{2} y=z, S q^{1} z=x^{2} \neq 0$, and $x^{4}=y^{2}=z^{2}=0$. This space $\mathrm{DI}(4)$ is an exotic 2 -adic finite loop space.

Received by the editors March 19, 1991.

1991 Mathematics Subject Classification. Primary 55P35; Secondary 55R40, 55P45.

This work was supported in part by the National Science Foundation. 
It is natural to ask about the realizability of other Dickson invariant algebras. Say that a space $Y$ is of type $B \mathrm{DI}(n)$ if $H^{*}\left(Y, \mathbf{F}_{2}\right)$ is isomorphic, as an algebra over the Steenrod algebra, to the algebra of rank $n$ mod 2 Dickson invariants [32]. Then $R P^{\infty}=B \mathrm{Z} / 2$ is of type $B \mathrm{DI}(1), B \mathrm{SO}(3)$ is of type $B \mathrm{DI}(2)$ and the classifying space $B \mathrm{G}_{2}$ of the exceptional Lie group $\mathrm{G}_{2}$ is of type $B \mathrm{DI}(3)$. It is known that no space of type $B \mathrm{DI}(n)$ can exist for $n \geq 6$ [31]. Lannes has recently used methods similar to ours to show the nonexistence of a space of type $B \mathrm{DI}(5)$, while [24] proves the stronger statement that an $H$-space "of type $\Omega B \mathrm{DI}(5)$ " does not exist (see also [18]). The construction of $B \mathrm{DI}(4)$ in this paper completes the determination of the set of integers $n$ for which a space of type $B \mathrm{DI}(n)$ exists.

One indication that $B \mathrm{DI}(4)$ might exist comes from Lie theory. If $G$ is a connected compact Lie group of rank $r$, then the Weyl group $W_{G}$ is a finite subgroup of $\mathrm{GL}(r, \mathbf{Q})$ generated by reflections, and the rational cohomology ring of $B G$ is naturally isomorphic to the ring of polynomial invariants of $W_{G}$. Finite reflection groups are relatively uncommon, and in fact $G$ is close to being determined by its Weyl group $W_{G}$. Let $\widehat{\mathbf{Z}}_{2}$ denote the ring of 2-adic integers. About ten years ago the second author observed that there exists a finite reflection subgroup $W_{\mathrm{DI}(4)}$ of $\mathrm{GL}\left(3, \mathbf{Q} \otimes \widehat{\mathbf{Z}}_{2}\right)$ such that the ring $\mathbf{Q} \otimes$ $H^{*}\left(B \mathrm{DI}(4), \widehat{\mathbf{Z}}_{2}\right)$ (which is easily seen to be a polynomial algebra on generators of dimensions 8,12 , and 28 ) is isomorphic to the ring of polynomial invariants of $W_{\mathrm{DI}(4)}$. In a sense this group $W_{\mathrm{DI}(4)}$ is a plausible "Weyl group" for a 2-adic finite loop space of type $\mathrm{DI}(4)$. The group $W_{\mathrm{DI}(4)}$ is isomorphic to $\mathbf{Z} / 2 \times \mathrm{GL}\left(3, \mathbf{F}_{2}\right)$ and arises as complex reflection group (number 24 on the list of [5]) whose reflection representation can be defined over a subfield of $\mathbf{C}$ which embeds in $\mathbf{Q} \otimes \widehat{\mathbf{Z}}_{2}$ [5, p. 431]. In 1980 there was no evident way to use the existence of $W_{\mathrm{DI}(4)}$ to construct $B \mathrm{DI}(4)$; even now we use the existence of $W_{\mathrm{DI}(4)}$ only in an indirect way $(\S 4)$ and make no explicit reference to the reflection properties of the group. In spite of this, the results of this paper suggest a link between $p$-adic finite loop spaces and $p$-adic reflection groups that lies deeper than the material in [9]. One might even conjecture on the basis of the tables in [5] that the classifying space of any connected 2-adic finite loop space is the product of the $\mathbf{F}_{2}$-completion of the classifying space of a connected compact Lie group with a number of copies of $B \mathrm{DI}(4)$.

1.2. The basic technique. Our present method of building $B \mathrm{DI}(4)$ depends on the ideas of $[20,17,13]$. The starting point is again the theory of Lie groups, but this time on the homotopy-theoretic side. Let $\mathscr{T}$ denote the category of topological spaces. Jackowski and McClure have proved the following remarkable result.

1.3. Proposition [17]. For any compact Lie group $G$ there exist

(1) a category $\mathbf{A}_{G}^{\mathrm{Lie}}$ whose objects are the (conjugacy classes of) nontrivial elementary abelian 2-subgroups of $G$,

(2) a functor $\alpha_{G}^{\mathrm{Lie}}:\left(\mathbf{A}_{G}^{\mathrm{Lie}}\right)^{\text {op }} \rightarrow \mathscr{T}$ which up to homotopy assigns to each such subgroup the classifying space of its centralizer in $G$, and

(3) a map holim $\alpha_{G}^{\text {Lie }} \rightarrow B G$ which is an isomorphism on $\mathbf{F}_{2}$ cohomology. 
Unless $G$ has a central element of order 2, this proposition provides a decomposition of $B G$ up to $\mathbf{F}_{2}$ cohomology as a generalized pushout of classifying spaces of proper subgroups of $G$. There is also an algebraic version of the above. Let $\mathscr{K}$ denote the category of unstable algebras over the mod 2 Steenrod algebra.

\subsection{Proposition [13, §2]. For any object $R$ of $\mathscr{K}$ there exist}

(1) a category $\mathbf{A}_{R}^{\text {alg }}$ in which an object is a pair $\left(V, f^{*}\right)$ consisting of a nontrivial elementary abelian 2-group $V$ together with (1.5) a suitable $\mathscr{K}$-map $f^{*}: R \rightarrow H^{*}\left(B V, \mathbf{F}_{2}\right)$,

(2) a Lannes functor $\alpha_{R}^{\mathrm{alg}}:\left(\mathbf{A}_{Y}^{\mathrm{alg}}\right)^{\mathrm{op}} \rightarrow \mathscr{K}$ which sends the pair $\left(V, f^{*}\right)$ to an algebra $T_{f^{*}}^{V}(R)$ (see 1.5), and

(3) a map $R \rightarrow \underset{\lim }{\longleftarrow} \alpha_{R}^{\mathrm{alg}}$ which in favorable cases is an isomorphism. Moreover if $R=H^{*}\left(B G, \mathbf{F}_{2}\right) \quad(G$ compact Lie $)$ then $[21,13]$ the categories $\mathbf{A}_{R}^{\mathrm{alg}}$ and $\mathbf{A}_{G}^{\mathrm{Lie}}$ are equivalent in such a way that the functors $\alpha_{R}^{\mathrm{alg}}$ and $H^{*}\left(\alpha_{G}^{\mathrm{Lie}}, \mathbf{F}_{2}\right)$ correspond.

Now let $Y$ be $B \mathrm{DI}(4)$ and let $R=H^{*}\left(Y, \mathbf{F}_{2}\right)$ be the rank 4 Dickson invariant algebra. It is easy to figure out the category $\mathbf{A}_{R}^{\text {alg }}$ (see 1.5) and for each object $\left(V, f^{*}\right)$ of $\mathbf{A}_{R}^{\mathrm{alg}}$ to compute the algebra $\alpha_{R}^{\mathrm{alg}}\left(V, f^{*}\right)$. It is natural to hope that $Y$ is sufficiently similar to the classifying space of a compact Lie group that something like the Jackowski-McClure decomposition might work for $Y$. With this as motivation, then, we construct by hand ( $(7)$ a functor $F:\left(\mathbf{A}_{R}^{\mathrm{alg}}\right)^{\text {op }} \rightarrow \mathscr{T}$ such that $H^{*}\left(F, \mathbf{F}_{2}\right)$ is naturally equivalent to $\alpha_{R}^{\mathrm{alg}}$, and verify by direct algebraic calculation (2.4) that the cohomology of the homotopy direct limit of this functor is the ring of rank 4 mod 2 Dickson invariants. We obtain $B \mathrm{DI}(4)$ as the $\mathbf{F}_{2}$-completion of this homotopy direct limit.

To build the functor $F$ we first $(\S 6)$ construct a parallel functor $\bar{F}$ with values in the homotopy category and then use the obstruction theory of [6] to lift $\bar{F}$ to a functor $F$ with values in the category of spaces. We are fortunate that this lifting problem is easy to solve once it is set up: all of the obstruction groups are zero (7.6).

1.5. The role of $\operatorname{Spin}(7)$. The construction of the above functor $\bar{F}$ takes up a large part of the paper and involves careful study of the Lie group Spin(7) and some of its subgroups. (Here $\operatorname{Spin}(7)$ is the connected double covering group of the special orthogonal group $\mathrm{SO}(7)$.) There is a simple explanation for this. Let $R=H^{*}\left(B \mathrm{DI}(4), \mathrm{F}_{2}\right)$ be the rank 4 Dickson invariant algebra. Because of 1.3 and 1.4 it is useful to refer to an object $A=\left(V, f^{*}\right) \in \mathbf{A}_{R}^{\text {alg }}$ with $\operatorname{rank}(V)=r$ as a rank $r$ elementary abelian 2-subgroup of $\mathrm{DI}(4)$ (taken up to conjugacy), and to interpret $\alpha_{R}^{\text {alg }}(A)$ as the cohomology of the classifying space of the centralizer of $A$ in $\mathrm{DI}(4)$. Explicitly, then, the elementary abelian 2-subgroups of $\mathrm{DI}(4)$ correspond to pairs $\left(V, f^{*}\right)$ where $V$ is an elementary abelian 2-group and $f^{*}: R \rightarrow H^{*}\left(B V, \mathbf{F}_{2}\right)$ is a $\mathscr{K}$-map which makes $H^{*}\left(B V, \mathbf{F}_{2}\right)$ into a finitely generated module over $R[13, \S 2]$. The following lemma (due to Lannes) explains how to enumerate these and calculate the values of the functor $\alpha_{R}^{\mathrm{alg}}$. 
We will let $T^{V}$ denote the $T$-functor of Lannes [19]; $T^{V}$ is the left adjoint on the category of unstable modules (or algebras) over the Steenrod algebra to the functor given by tensoring with $H^{*}\left(B V, \mathbf{F}_{2}\right)$. If $X$ is a space the algebra $T^{V}\left(H^{*}\left(X, \mathbf{F}_{2}\right)\right)$ is closely related to the $\mathbf{F}_{2}$ cohomology algebra of the space of maps from $B V$ to $X$. Recall [11] that to any object $S \in \mathscr{K}$ and map $f^{*}: S \rightarrow H^{*}\left(B V, \mathbf{F}_{2}\right)$ there is associated a quotient object $T_{f^{*}}^{V}(S)$ of $T^{V}(S)$; if $S=H^{*}\left(X, \mathbf{F}_{2}\right)$ then $T_{f^{*}}^{V}(S)$ is closely related to the $\mathbf{F}_{2}$ cohomology ring of the space of maps $B V \rightarrow X$ inducing $f^{*}$ on cohomology. (Note that in [13] the algebra $T_{f^{*}}^{V}(S)$ is denoted $T(V, S)_{f}$.)

1.6. Lemma. Let $E$ be an elementary abelian 2-group, $\Gamma$ a subgroup of $\operatorname{Aut}(E)$, and $S$ the unstable algebra over the Steenrod algebra given by the fixed point set $H^{*}\left(B E, \mathbf{F}_{2}\right)^{\Gamma}$. Let $V$ be an elementary abelian 2-group.

(1) The map which assigns to each homomorphism $f: V \rightarrow E$ the induced composite $S \rightarrow H^{*}\left(B E, \mathbf{F}_{2}\right) \stackrel{f^{*}}{\rightarrow} H^{*}\left(B V, \mathbf{F}_{2}\right)$ gives a bijection between $\mathscr{K}$-maps $f^{*}: S \rightarrow H^{*}\left(B V, \mathbf{F}_{2}\right)$ and $\Gamma$-orbits of homomorphisms $f: V \rightarrow E$.

(2) $A$ map $f^{*}: S \rightarrow H^{*}\left(B V, \mathbf{F}_{2}\right)$ makes $H^{*}\left(B V, \mathbf{F}_{2}\right)$ into a finitely generated $S$ module iff the corresponding (1) homomorphism $f: V \rightarrow E$ is a monomorphism.

(3) If $f^{*}: S \rightarrow H^{*}\left(B V, \mathbf{F}_{2}\right)$ corresponds to $f: V \rightarrow E$, then $T_{f^{*}}^{V}(S)$ is naturally isomorphic to $H^{*}\left(B E, \mathbf{F}_{2}\right)^{\Gamma_{f}}$, where $\Gamma_{f} \subset \Gamma$ is the subgroup of elements which pointwise fux the image of $f$.

Proof. By exactness of the functor $T^{V}$ [21] there is an isomorphism

$$
T^{V}(S)=T^{V}\left(H^{*}\left(B E, \mathbf{F}_{2}\right)^{\Gamma}\right) \cong T^{V}\left(H^{*}\left(B E, \mathbf{F}_{2}\right)\right)^{\Gamma}
$$

Moreover, the algebra $T^{V}\left(H^{*}\left(B E, \mathbf{F}_{2}\right)\right)$ is isomorphic [21, 3.4.5] as a $\Gamma$-object to the tensor product $H^{0}\left(\operatorname{Hom}(V, E), \mathbf{F}_{2}\right) \otimes H^{*}\left(B E, \mathbf{F}_{2}\right)$ with the diagonal $\Gamma$ action (the first factor here is the zero-dimensional cohomology of the discrete space $\operatorname{Hom}(V, E)$; the tensor product itself in fact gives the cohomology of the space of maps $B V \rightarrow B E$ ). Parts (1) and (3) follow from the definition of $T_{f^{*}}^{V}$ and inspection. Part (2) can be obtained for instance from [13, proof of 4.4].

Since the group $\Gamma=\operatorname{GL}\left(4, \mathbf{F}_{2}\right)$ acts transitively on the subspaces of $(Z / 2)^{4}$ of any given dimension, it follows that up to conjugacy there is only one rank $i$ elementary abelian 2-subgroup $A_{i}$ of $\mathrm{DI}(4)$ for each $i=1, \ldots, 4$. Moreover, the algebra $\alpha_{R}^{\text {alg }}\left(A_{i}\right)$ is isomorphic to the fixed point set of the action of $\Gamma_{i}$ on $H^{*}\left((B Z / 2)^{4}, \mathbf{F}_{2}\right)$, where $\Gamma_{i} \subset \mathrm{GL}\left(4, \mathbf{F}_{2}\right)$ is the set of matrices which agree with the identity in the first $i$ columns. A study of morphisms [13, §2] now implies that the category $\mathbf{A}_{R}^{\text {alg }}$ is equivalent to the category $\mathbf{A}$ of $\S 2$ and that the functor $\alpha_{R}^{\mathrm{alg}}$ is equivalent to the functor $\alpha_{R}$ of 2.3. The category $\mathbf{A}$ has 
four objects and the following shape

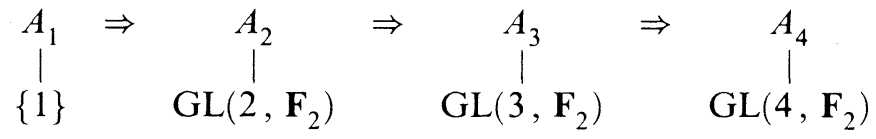

where under each object is its monoid (in this case group) of self maps and " $\Rightarrow$ " stands for an appropriate set of morphisms. There are no morphisms $A_{i} \rightarrow A_{j}$ for $i>j$ and for $i \leq j$ the group $\mathrm{GL}\left(j, \mathbf{F}_{2}\right)=\operatorname{Aut}\left(A_{j}\right)$ acts transitively on the set of morphisms $A_{i} \rightarrow A_{j}$.

This calculation, though, reveals something perhaps unexpected: the cohomology ring $\alpha_{R}^{\mathrm{alg}}\left(A_{1}\right)$ of the classifying space of the centralizer of the essentially unique rank 1 elementary abelian 2-subgroup of $\mathrm{DI}(4)$ (the cohomology ring calculated as $H^{*}\left((B Z / 2)^{4}, \mathbf{F}_{2}\right)^{\Gamma_{1}}$ above) is exactly (3.11) the $\mathbf{F}_{2}$ cohomology ring of $B \operatorname{Spin}(7)$ ! Encouraged by this, we surmise that this centralizer is Spin(7) and begin the construction of $\bar{F}$ by declaring $\bar{F}\left(A_{1}\right)$ to be the $\mathbf{F}_{2}$ completion of $B \operatorname{Spin}(7)$. It then stands to reason that the centralizer in $\operatorname{DI}(4)$ of a rank 2 elementary abelian 2-subgroup $A$, say, should be the same as the centralizer of $A$ inside the centralizer within DI(4) of any rank 1 subgroup of $A$, i.e., the same as the centralizer of $A$ within Spin(7). This can be verified cohomologically by combining $1.3,1.4$ and 1.6; verification comes down to observing that the fixed point set of the action of the group $\Gamma_{i}(2 \leq i \leq 4)$ on $H^{*}\left((B \mathbf{Z} / 2)^{4}, \mathbf{F}_{2}\right)$ does not depend on whether $\Gamma_{i}$ is encountered as a subgroup of $\Gamma_{1}$ or as a subgroup of $\mathrm{GL}\left(4, \mathbf{F}_{2}\right)$. The conclusion is (3.13) that for $i=2,3,4$ the algebra $\alpha_{R}^{\text {alg }}\left(A_{i}\right)$ is isomorphic to $H^{*}\left(B \mathscr{C}\left(E_{i}\right), \mathbf{F}_{2}\right)$, where $\mathscr{C}\left(E_{i}\right)$ is the centralizer in $\operatorname{Spin}(7)$ of a rank $i$ elementary abelian 2-group $E_{i}$ containing the center of $\operatorname{Spin}(7)$. This is evidence enough for us to declare $\bar{F}\left(A_{i}\right)$ to be the $\mathbf{F}_{2}$-completion of $B \mathscr{C}\left(E_{i}\right)$.

1.7. Final ingredients. Once we have settled on the spaces which the functor $\bar{F}$ will take on as its values, we have to look for homotopy classes of maps between these spaces satisfying the relations described in 2.1 . The basic maps $\bar{F}\left(A_{i}\right) \rightarrow \bar{F}\left(A_{j}\right) \quad(i>j)$ arise from the inclusion of one subgroup of $\operatorname{Spin}(7)$ in another. No nonidentify self-maps of $\bar{F}\left(A_{1}\right)$ are required, and the necessary self-maps of $\bar{F}\left(A_{4}\right)$ are easy to construct, since this space is just $(B \mathbf{Z} / 2)^{4}$. For $i=2$ and $i=3$, though, it turns out that inner automorphisms of $\operatorname{Spin}(7)$ provide actions of a certain matrix group $(\S 2) P(1, i-1) \subset \mathrm{GL}\left(i, \mathbf{F}_{2}\right)$ on $\bar{F}\left(A_{i}\right)$, whereas the existence of $\bar{F}$ as a functor requires an action of the full group $\mathrm{GL}\left(i, \mathbf{F}_{2}\right)$ on this space. The case $i=2$ of this problem is solved in $\S 5$, and the case $i=3$ in $\S 4$ (this last case is particularly interesting because it involves constructing what is essentially a 2-adic integral form of the basic reflection representation of $\left.W_{\mathrm{DI}(4)}\right)$. A significant amount of effort goes into being explicit enough about these solutions that it is possible to verify concretely that the necessary diagrams commute up to homotopy and that they have the correct cohomological properties (see $\S 6$ ). 
In the end our diagram looks like this:

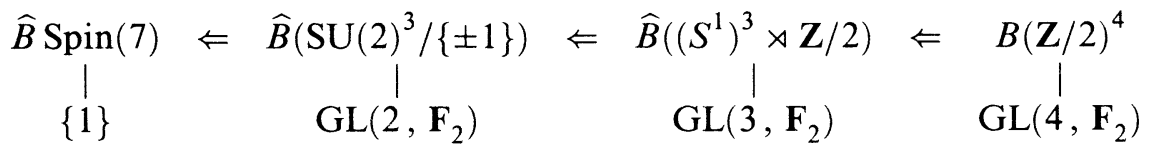

where " $\widehat{B} X$ " denotes the $\mathbf{F}_{2}$ completion of “ $B X$ ". (See $\S 3$ for detailed descriptions of the above groups.) Of course the spaces in the above diagram exist integrally, but the maps we use exist only after $\mathbf{F}_{2}$ completion.

Finally, we note that the conflict between Theorem 1.1 and the nonrealizability results of [25] has been resolved in [23].

Lie-like properties. The construction of $B \mathrm{DI}(4)$ given in this paper begins with the hypothesis that this space has properties similar to those of the classifying space of a compact Lie group. Theorem 1.8, whose proof is computational but routine, points out a few striking Lie-like properties that follow from the construction.

1.8. Theorem. The space $B \mathrm{DI}(4)$ has the following properties:

(1) There is a map $f: B \operatorname{Spin}(7) \rightarrow B \mathrm{DI}(4)$ such that the homotopy fibre $\mathrm{DI}(4) / \operatorname{Spin}(7)$ has $\mathbf{F}_{2}$ cohomology which is finite, concentrated in even degrees, and has Euler characteristic 24.

Let $B T \rightarrow B \operatorname{Spin}(7)$ be induced by the inclusion of the (rank 3) maximal torus, let $g$ denote the composite $B T \rightarrow B \operatorname{Spin}(7) \rightarrow B \mathrm{DI}(4)$ and let $\mathrm{DI}(4) / T$ denote the homotopy fibre of $g$.

(2) $H^{*}\left(\mathrm{DI}(4) / T, \mathbf{F}_{2}\right)$ is finite, concentrated in even degrees, and has Euler characteristic $336=\left|W_{\mathrm{DI}(4)}\right|$.

(3) The space of self-equivalences of $\widehat{B} T$ (see 1.9) over B DI(4) is homotopically discrete and has $W_{\mathrm{DI}(4)}$ as its group of components $(c f .[9,2.8])$.

The space $\mathrm{DI}(4) / \operatorname{Spin}(7)$ is perhaps an exotic symmetric space at the prime 2. The space $\mathrm{DI}(4) / T$ is the flag manifold (at the prime 2 ) corresponding to $\mathrm{DI}(4)$.

1.9. Notation and terminology. We will follow the standard convention that composition of maps between sets, groups, etc. works from right to left: a composite function $f \cdot g$ or $f g$ is defined by the formula $f \cdot g(x)=f(g(x))$. In particular, if $X$ is a set, group, etc. the monoid $\operatorname{Hom}(X, X)$ acts in a natural way on $X$ from the left.

Unless otherwise specified, cohomology is taken with coefficients in the field $\mathbf{F}_{2}$ of two elements. If $X$ and $Y$ are spaces then $\operatorname{MAP}(X, Y)$ denotes the function space of maps from $X$ to $Y$; the component containing a particular map or homotopy class $f$ is $\operatorname{MAP}(X, Y)_{f}$. The space $\operatorname{EMB}(X, Y)$ is the subspace of $\operatorname{MAP}(X, Y)$ containing maps with respect to which $H^{*} X$ is a finitely generated module over $H^{*} Y ; \operatorname{AUT}(X)$ is the topological monoid of self-homotopy equivalences of $X$ with identity component $\operatorname{AUT}(X)_{1}$. The symbol $\widehat{B} G$ will stand for the $\mathbf{F}_{2}$-completion of the classifying space $B G$.

The center of a (Lie) group $G$ is denoted $\mathscr{Z}(G)$; if $G$ is given as a subgroup of some ambient group $H$ then $\mathscr{C}(G)$ and $\mathscr{N}(G)$ denote respectively the 
centralizer and normalizer of $G$ in $H$. For general compact Lie groups $G$ and $H$ we will let $\operatorname{Emb}(G, H)$ stand for the set of $H$-conjugacy classes of group monomorphisms $G \rightarrow H$; note $[29,2.4]$ that there are natural maps $\operatorname{Emb}(G, H) \rightarrow \pi_{0} \operatorname{Eмв}(B G, B H) \rightarrow \pi_{0} \operatorname{EMB}(\widehat{B} G, \widehat{B} H)$.

The symbol $\Sigma_{n}$ will stand for the symmetric group of degree $n$, i.e., the group of automorphisms of the set $\{1, \ldots, n\}$. Suppose that $G$ is a (topological) group or monoid. The wreath product $\Sigma_{n} l G$ is the space $\Sigma_{n} \times G^{n}$ with multiplication determined by the formula

$$
\left[g_{1}, \ldots, g_{n}\right] \cdot \sigma=\sigma \cdot\left[g_{\sigma(1)}, \ldots, g_{\sigma(n)}\right],
$$

where $\sigma \in \Sigma_{n}$ and $\left[g_{1}, \ldots, g_{n}\right]$ belongs to the product monoid $G^{n}$.

\section{THE BASIC CATEgORY}

In this section we describe the basic index category $\mathbf{A}$ which is used in the paper; then we construct and study the algebraic diagram over $\mathbf{A}$ which we intend eventually to realize geometrically.

Let $\mathbf{A}$ be the category whose objects are the $\mathbf{F}_{2}$ vector spaces $(\mathbf{Z} / 2)^{i}, i=$ $1, \ldots, 4$, and whose morphisms are vector space monomorphisms. Give the object $(\mathbf{Z} / 2)^{i}$ (denoted $A_{i}$ ) the standard ordered basis $e_{i}(j), j=1, \ldots, i$, where $e_{i}(j)$ is zero except in the $j$ th coordinate. It is clear that $\operatorname{Hom}_{\mathbf{A}}\left(A_{i}, A_{j}\right)$ is empty if $i>j$, and that (in view of the chosen basis for $A_{i}$ ) the group $\operatorname{Hom}_{\mathbf{A}}\left(A_{i}, A_{i}\right)$ is $\mathrm{GL}\left(i, \mathbf{F}_{2}\right)$. We will think of the elements of $A_{i}$ as column vectors, so that as usual (1.9) the group $\mathrm{GL}\left(i, \mathbf{F}_{2}\right)$ acts on the elements of $A_{i}$ from the left.

There are standard embeddings $f_{i}: A_{i} \rightarrow A_{i+1}, i=1, \ldots, 3$, in A determined by the formula $f_{i}\left(e_{i}(j)\right)=e_{i+1}(j)$. Let $P(i, j)$ denote the subgroup of $\mathrm{GL}\left(i+j, \mathbf{F}_{2}\right)$ consisting of matrices of the form

$$
\left(\begin{array}{cc}
M_{11} & M_{12} \\
0 & M_{22}
\end{array}\right)
$$

where $M_{11} \in \mathrm{GL}\left(i, \mathbf{F}_{2}\right)$, and let $r_{i}: P(i, j) \rightarrow \mathrm{GL}\left(i, \mathbf{F}_{2}\right)$ be the homomorphism which sends the above element of $P(i, j)$ to the matrix $M_{11}$. The group $P(i, j)$ is the subgroup of $\mathrm{GL}\left(i+j, \mathbf{F}_{2}\right)$ given by transformations which carry to itself the subspace of $A_{i+j}$ generated by the first $i$ standard basis elements.

The following lemma is an elementary calculation.

2.1. Lemma. The category $\mathbf{A}$ is generated by the groups

$$
\mathrm{GL}\left(i, \mathbf{F}_{2}\right)=\operatorname{Hom}_{\mathbf{A}}\left(A_{i}, A_{i}\right), \quad i=1, \ldots, 4,
$$

and the maps $f_{i}: A_{i} \rightarrow A_{i+1}, i=1, \ldots, 3$, subject to the following relations:

$$
\begin{aligned}
& g \cdot f_{1}=f_{1} \cdot r_{1}(g), \quad g \in P(1,1), \\
& g \cdot f_{2}=f_{2} \cdot r_{2}(g), \quad g \in P(2,1), \\
& g \cdot f_{3}=f_{3} \cdot r_{3}(g), \quad g \in P(3,1), \\
& g \cdot f_{2} \cdot f_{1}=f_{2} \cdot f_{1} \cdot r_{1}(g), \quad g \in P(1,2), \\
& g \cdot f_{3} \cdot f_{2}=f_{3} \cdot f_{2} \cdot r_{2}(g), \quad g \in P(2,2), \\
& g \cdot f_{3} \cdot f_{2} \cdot f_{1}=f_{3} \cdot f_{2} \cdot f_{1} \cdot r_{1}(g), \quad g \in P(1,3) .
\end{aligned}
$$


2.2. Remark. Lemma 2.1 implies that in order to construct a functor $F: \mathbf{A} \rightarrow$ $\mathbf{C}$ for some category $\mathbf{C}$, it is enough to find objects $F\left(A_{i}\right)=C_{i}, i=1, \ldots, 4$, of $\mathbf{C}$, homomorphisms $\mathrm{GL}\left(i, \mathbf{F}_{2}\right) \rightarrow \operatorname{Hom}_{\mathbf{C}}\left(C_{i}, C_{i}\right)$ and maps $F\left(f_{i}\right): C_{i} \rightarrow$ $C_{i+1}, i=1, \ldots, 3$, such that relations parallel to $2.1(1)-2.1(6)$ are satisfied.

2.3. A cohomology diagram. Now let $V$ stand for the elementary abelian group $(\mathrm{Z} / 2)^{4}$. The natural (left) action of $\operatorname{Aut}(V)$ on $V$ induces a right action of Aut $(V)$ on the cohomology ring $H^{*} B V$. The ring of elements fixed under this action is the algebra $R$ of rank $4 \bmod 2$ Dickson invariants [32].

If $A$ is an object of $\mathbf{A}$ let $\widetilde{\alpha}_{R}(A)=H^{*} \operatorname{EMB}(B A, B V)$; this gives a functor $\widetilde{\alpha}_{R}$ from $\mathbf{A}$ to the category of right $\operatorname{Aut}(V)$-objects in the category $\mathscr{K}$ (1.2). Note (cf. proof of 1.6) that $\widetilde{\alpha}_{R}(A)$ is isomorphic to the tensor product $H^{0} \operatorname{Emb}(A, V) \otimes H^{*} B V$ with the diagonal action of $\operatorname{Aut}(V)$. Let $\alpha_{R}$ be the fixed point subfunctor of $\widetilde{\alpha}_{R}$, so that for each object $A_{i}$ of $\mathbf{A}, \alpha_{R}\left(A_{i}\right)=$ $\widetilde{\alpha}_{R}\left(A_{i}\right)^{\mathrm{Aut}(V)}$.

2.4. Proposition. There is a natural isomorphism $R \cong \lim \alpha_{R}$. The higher derived functors $\lim ^{i} \alpha_{R}$ vanish for $i>0$.

Proof. Let $\Gamma=\operatorname{Aut}(V)$ and let $M$ be the right $\operatorname{Aut}(V)$ module $S=H^{*} B V$ treated as a left $\Gamma$ module via the inversion map of $\operatorname{Aut}(V)$. The category $\mathbf{A}$ is equivalent to the category $\mathbf{A}_{\Gamma}$ of $\S 8$ in such a way that $\alpha_{R}$ corresponds to the functor $\alpha_{\Gamma, M}$; Proposition 8.1 thus gives the desired result. (Note that in this special case, in which $\Gamma=\operatorname{Aut}(V)$ is as large as possible, the category $\mathbf{A}_{\Gamma}$ is equivalent to the category $\mathbf{B}$ of 8.3 in such a way that the functor $\alpha_{\Gamma, M}$ is equivalent to $\beta_{\Gamma, M}$.)

Proposition 2.4 also follows from the main theorem of [13], since in fact the functor $\alpha_{R}$ of 2.4 is naturally equivalent to the functor $\alpha_{R}$ of $[13,1.5]$.

Suppose that $F: \mathbf{A}^{\text {op }} \rightarrow \mathscr{T}$ is a functor, where $\mathscr{T}$ is the category of topological spaces. According to [3, XII, 5.8] there is a spectral sequence for the cohomology of the homotopy direct limit holim $F$ which has the form

$$
\left\{E_{2}^{p, q}=\lim p H^{q}(F)\right\} \Rightarrow H^{p+q}(\operatorname{holim} F) .
$$

If $H^{*}(F) \cong \alpha_{R}$, then by 2.4 the spectral sequence collapses into the isomorphism $H^{*}($ holim $F) \cong R$ and by [3, VII, 3.2] the $\mathbf{F}_{2}$-completion of holim $F$ is an $\mathbf{F}_{2}$-complete space with cohomology given by $R$. In this way the following result implies Theorem 1.1 .

2.5. Theorem. There exists a functor $F: \mathbf{A}^{\mathrm{op}} \rightarrow \mathscr{T}$ such that $H^{*}(F)$ is naturally equivalent to $\alpha_{R}$.

For the rest of the paper we will concentrate on proving 2.5.

\section{Subgroups of $\operatorname{Spin}(7)$}

In $\S 2$ we described a very small category $\mathbf{A}$ which up to equivalence should be the category of conjugacy classes of elementary abelian subgroups (1.5) of 
$\mathrm{DI}(4)$, and we reduced the problem of constructing $B \mathrm{DI}(4)$ to that of constructing a suitable functor $F: \mathbf{A}^{\text {op }} \rightarrow \mathscr{T}$. As explained in 1.5 , the spaces which the functor $F$ will take on as its values will be of the form $\widehat{B} K$, where $K$ is the centralizer in $\operatorname{Spin}(7)$ of an elementary abelian 2-group containing the center of $\operatorname{Spin}(7)$. In this section we take the first steps toward constructing $F$ (or its homotopy version $\bar{F}$ ) by identifying (3.2) within $\operatorname{Spin}(7)$ the appropriate elementary abelian 2-subgroups $E_{i}(i=1, \ldots, 4)$ and collecting needed information about

(1) the centralizers $\mathscr{C}\left(E_{i}\right)$ of the groups $E_{i}$ (3.3),

(2) the normalizers $\mathscr{N}\left(\mathscr{C}\left(E_{i}\right)\right)$ of the groups $\mathscr{C}\left(E_{i}\right)$ (3.5), and

(3) the cohomology rings $H^{*} B \mathscr{C}\left(E_{i}\right)$ (3.8).

The normalizers (2) determine certain key self-equivalences of the spaces $\widehat{B} \mathscr{C}\left(E_{i}\right)$ (the "internal Spin actions" of $\S 6$ ). In order for us to prove that the functor $\bar{F}$ constructed in $\S 6$ has the correct cohomological behavior, it is necessary to understand the cohomology rings (3) in a functorial, geometric way (3.13).

Let $\bar{T}$ be the maximal torus in $\mathrm{SO}(7)$ given by block matrices

$$
\left(\begin{array}{cccc}
M_{1} & 0 & 0 & 0 \\
0 & M_{2} & 0 & 0 \\
0 & 0 & M_{3} & 0 \\
0 & 0 & 0 & 1
\end{array}\right)
$$

where each $M_{i}$ is of the form

$$
\left(\begin{array}{cc}
\cos \theta_{i} & -\sin \theta_{i} \\
\sin \theta_{i} & \cos \theta_{i}
\end{array}\right) .
$$

The Lie algebra of $\bar{T}$ is a subalgebra of the Lie algebra $\operatorname{so(7)}$ of $7 \times 7$ skewsymmetric real matrices. Let $\bar{L}$ denote the kernel of the exponential map on the Lie algebra of $\bar{T} ; \bar{L}$ is a free $\mathbf{Z}$ module of $\operatorname{rank} 3$ with basis $\bar{\ell}_{i}$ $(i=1, \ldots, 3)$, where

$$
\bar{\ell}_{i}=\left(\begin{array}{cccc}
M_{1}^{i} & 0 & 0 & 0 \\
0 & M_{2}^{i} & 0 & 0 \\
0 & 0 & M_{3}^{i} & 0 \\
0 & 0 & 0 & 0
\end{array}\right)
$$

with $M_{j}^{i}=\left(\begin{array}{cc}0 & -2 \pi \\ 2 \pi & 0\end{array}\right)$ if $i=j$ and $M_{j}^{i}=0$ otherwise. Let $T$ be the torus in Spin(7) which is the inverse image of $\bar{T}$ and $L$ the kernel of the exponential map on the Lie algebra of $T$. The natural map $L \rightarrow \bar{L}$ exhibits $L$ as the index two subgroup of $\bar{L}$ consisting of elements $n_{1} \bar{\ell}_{1}+n_{2} \bar{\ell}_{2}+n_{3} \bar{\ell}_{3}$ such that $n_{1}+n_{2}+n_{3}$ is even; this is spanned by the set $\left\{\ell_{1}, \ell_{2}, \ell_{3}\right\}$ where $\ell_{1}=2 \bar{\ell}_{1}$, $\ell_{2}=\bar{\ell}_{1}-\bar{\ell}_{2}$, and $\ell_{3}=\bar{\ell}_{1}+\bar{\ell}_{3}$. The group $L$ is naturally isomorphic to the fundamental group $\pi_{1} T$.

Recall that the map $B \operatorname{Spin}(7) \rightarrow B \operatorname{SO}(7)$ is up to homotopy a principal fibration with fibre $K(\mathbf{Z} / 2,1)$ classified by the second Steifel-Whitney class $w_{2} \in H^{2} B \mathrm{SO}(7)$. We will implicitly use the following lemma several times. 
3.1. Lemma. Let $\bar{V}$ be an elementary abelian 2-subgroup of $\mathrm{SO}(7), V$ its inverse image in $\operatorname{Spin}(7)$, and $w_{2}(\bar{V}) \in H^{2} B \bar{V}$ the restriction of $w_{2}$. Then $V$ is an abelian group iff $w_{2}(\bar{V})$ is the square of a one-dimensional class and an elementary abelian group iff $w_{2}(\bar{V})=0$.

Remark. Often the best way to compute the class $w_{2}(\bar{V})$ of 3.1 is to express the representation $\bar{V} \rightarrow \mathrm{SO}(7)$ as a sum of one-dimensional representations of $\bar{V}$.

3.2. Elementary abelian subgroups of $\operatorname{Spin}(7)$. We will let $\bar{E}$ be the rank three elementary abelian 2-subgroup of $\mathrm{SO}(7)$ generated by the matrices

$$
\operatorname{diag}(----+++), \quad \operatorname{diag}(--++--+), \quad \operatorname{diag}(-+-+-+-),
$$

where $\operatorname{diag}(\cdots)$ indicates a diagonal matrix with the specified pattern of \pm 1 entries. By 3.1 the inverse image $E_{4}$ of $\bar{E}$ in $\operatorname{Spin}(7)$ is a rank 4 elementary abelian 2-group, and it is clear that $E_{4}$ contains a rank 3 elementary abelian subgroup $E_{3}$ generated by the elements of order 2 in the torus $T$. The exponential exact sequence $L \rightarrow L \otimes \mathbf{R} \rightarrow T$ shows that $E_{3}$ is naturally isomorphic to $\left(\frac{1}{2} L\right) / L \cong L / 2 L$; under this isomorphism the basis $\left\{\ell_{1}, \ell_{2}, \ell_{3}\right\}$ for $L$ provides a basis for $E_{3}$. Let $E_{2}$ and $E_{1}$ denote the (based) subgroups of $E_{3}$ corresponding to the subgroups of $L / 2 L$ generated respectively by $\left\{\ell_{1}, \ell_{2}\right\}$ and $\left\{\ell_{1}\right\}$. The group $E_{1}$ is the center of $\operatorname{Spin}(7)$, i.e., the kernel of the epimorphism $\operatorname{Spin}(7) \rightarrow \mathrm{SO}(7)$.

3.3. Centralizers of elementary abelian subgroups. We need to describe the centralizers of the above groups very explicitly (see 1.5). Let $Q$ stand for the multiplicative group of unit quaternions; this is isomorphic to the special unitary group $\mathrm{SU}(2)$. The center of $Q$ is $\{ \pm 1\}$, and we will use $Q_{\mathrm{red}}^{n}$ to stand for the quotient of $Q^{n}$ by the diagonal subgroup generated by $(-1, \ldots,-1)$. Let $T_{\text {ext }}^{n}$ denote the semidirect product of the $n$-torus $(\mathbf{R} / \mathbf{Z})^{n}$ with $\{ \pm 1\}$, where the generator of $\{ \pm 1\}$ acts on $(\mathbf{R} / \mathbf{Z})^{n}$ by inversion. Recall (1.9) that $\mathscr{C}\left(E_{i}\right)$ (resp. $\mathscr{N}\left(E_{i}\right)$ ) is the centralizer (resp. normalizer) in $\operatorname{Spin}(7)$ of $E_{i}$.

3.4. Lemma. The groups $\mathscr{C}\left(E_{i}\right), i=1, \ldots, 4$, are isomorphic respectively to $\operatorname{Spin}(7), Q_{\mathrm{red}}^{3}, T_{\mathrm{ext}}^{3}$, and $E_{4}$. The quotient group $\mathcal{N}\left(E_{4}\right) / \mathscr{C}\left(E_{4}\right)$ has order $8 \cdot\left|\mathrm{GL}\left(3, \mathbf{F}_{2}\right)\right|$.

Proof. We will use the fact that if $K \subset \operatorname{Spin}(7)$ is the inverse image of the subgroup $\bar{K} \subset \operatorname{SO}(7)$, then the normalizer of $K$ in $\operatorname{Spin}(7)$ is the inverse image of the normalizer of $\bar{K}$. Case $i=1$ of the lemma is clear. For $i=2$, note that the image of $E_{2}$ in $\mathrm{SO}(7)$ is the group $\bar{E}_{2}$ of order 2 generated by $\operatorname{diag}(---+++)$. The normalizer $\mathscr{N}\left(\bar{E}_{2}\right)$ of $\bar{E}_{2}$ in $\mathrm{SO}(7)$ is a twocomponent group with identity component $\mathrm{SO}(4) \times \mathrm{SO}(3)$; one element in the nonidentity component is $\operatorname{diag}(---+++-)$. By $3.1, \mathscr{C}\left(E_{2}\right)$ is the inverse image in $\operatorname{Spin}(7)$ of the identity component of $\mathscr{N}\left(E_{2}\right)$, and it is easy to check that this inverse image, as a two-fold covering group of $\mathrm{SO}(4) \times \mathrm{SO}(3) \cong$ $Q_{\text {red }}^{2} \times Q_{\text {red }}^{1}$, has the indicated form.

For $i=3$ observe that the image $\bar{E}_{3}$ of $E_{3}$ in $\mathrm{SO}(7)$ is the group generated by $\bar{E}_{2}$ and $\operatorname{diag}(--++--+)$; the representation $\bar{E}_{3} \rightarrow \mathrm{SO}(7)$ is the sum 
of the trivial one-dimensional representation of $\bar{E}_{3}$ with two copies of each nontrivial one-dimensional representation. The centralizer $\mathscr{C}\left(\bar{E}_{3}\right)$ of $\bar{E}_{3}$ in $\mathrm{SO}(7)$ is a group isomorphic to $\mathrm{O}(2)^{3}$ generated by the torus $\bar{T}$ and the rank 6 elementary abelian group $D$ of diagonal matrices. A check with 3.1 shows that $\mathscr{C}\left(E_{3}\right)$ is generated by the torus $T$ and the inverse image in $\operatorname{Spin}(7)$ of $\operatorname{diag}(-+-+-+-)$.

For $i=4$, observe that the image $\bar{E}_{4}$ of $E_{4}$ in $\operatorname{SO}(7)$ is generated by $\bar{E}_{3}$ and $\operatorname{diag}(-+-+-+-)$; the representation $\bar{E}_{4} \rightarrow \mathrm{SO}(7)$ is the sum of all seven nontrivial one-dimensional representations of $\bar{E}_{4}$. The centralizer $\mathscr{C}\left(\bar{E}_{4}\right)$ is generated by $\bar{E}_{4}$ and the group $D$ above; the quotient $\mathscr{N}\left(\bar{E}_{4}\right) / \mathscr{C}\left(\bar{E}_{4}\right)$ is isomorphic in its conjugation action on $\bar{E}_{4}$ to $\operatorname{Aut}\left(\bar{E}_{4}\right)$. It is now easy to see that $E_{4}$ is its own centralizer in $\operatorname{Spin}(7)$, since it is clear from the above that $E_{4}$ is its own centralizer in $\mathscr{C}\left(E_{3}\right)$. The desired result follows.

3.5. Normalizers of centralizers. We now study the normalizers in $\operatorname{Spin}(7)$ of each one of above centralizers. This is important to us because (3.6) the normalizers determine which self homotopy equivalences of the classifying spaces of the centralizers are realized by conjugations in $\operatorname{Spin}(7)$. In the end (see 1.7) we will have to augment these particular self homotopy equivalences by others in order to construct the functor $\bar{F}$, but it is necessary $(\S 6)$ in verifying the commutative diagrams that constitute $\bar{F}$ to recognize that certain equivalences are internal to $\operatorname{Spin}(7)$. We approach the problem of naming the equivalences (3.7) by labeling them with integral matrices (see 5.5 and the proof of 6.1 for an indication of how effective this is).

Note that for $i=1, \ldots, 4$ the group $E_{i}$ is the center $\mathscr{Z}\left(\mathscr{C}\left(E_{i}\right)\right)$, so that the normalizer $\mathscr{N}\left(E_{i}\right)$ of $E_{i}$ in $\operatorname{Spin}(7)$ is also the normalizer $\mathscr{N}\left(\mathscr{C}\left(E_{i}\right)\right)$ of $\mathscr{C}\left(E_{i}\right)$ in $\operatorname{Spin}(7)$. For $i=1,2,3$ let $W_{i}$ be the Weyl group of $\mathscr{C}\left(E_{i}\right)$ (i.e., the quotient by $T$ of the normalizer of $T$ in $\left.\mathscr{C}\left(E_{i}\right)\right)$, and let $W_{i}^{+}$be the quotient by $T$ of the normalizer of $T$ in $\mathscr{N}\left(E_{i}\right)$. The groups $W_{i}$ and $W_{i}^{+}$ are all in a natural way subgroups of the Weyl group $W_{1}$ of $\operatorname{Spin}(7)$; in fact there are inclusions $W_{3} \subset W_{2} \subset W_{2}^{+} \subset W_{3}^{+}=W_{1}=W_{1}^{+}$.

3.6. Remark. Since inner automorphisms of a group $G$ act trivially up to homotopy on $B G$, there are natural homotopy actions of $W_{i}^{+} / W_{i}$ on $B \mathscr{C}\left(E_{i}\right)$, $i=2,3$.

Given the chosen basis $\left\{\ell_{1}, \ell_{2}, \ell_{3}\right\}$ for $L$, the conjugation action of $W_{1}$ on $T$ provides a homomorphism $\rho_{W}: W_{1} \rightarrow \mathrm{GL}(3, \mathbf{Z})$. Let $\mu: \mathrm{GL}(3, \mathbf{Z}) \rightarrow$ $\mathrm{GL}(3, \mathbf{F})$ stand for reduction $\bmod 2$ and $\operatorname{det}: \mathrm{GL}(3, \mathbf{Z}) \rightarrow\{ \pm 1\}$ for the determinant map. Recall from $\S 2$ the definition of the subgroup $P(1,2)$ of $\mathrm{GL}\left(3, \mathbf{F}_{2}\right)$. Denote by $U(2,1)$ the subgroup of $P(2,1)$ consisting of matrices which agree with the identity except in the last column and by $U \subset \operatorname{GL}\left(3, \mathbf{F}_{2}\right)$ the group of upper-triangular matrices.

3.7. Lemma. The product map $\varphi=\left(\mu \cdot \rho_{W}\right.$, det $\left.\cdot \rho_{W}\right)$ induces isomorphism $W_{1} \cong P(1,2) \times\{ \pm 1\}, W_{2}^{+} \cong U \times\{ \pm 1\}, W_{2} \cong U(2,1) \times\{ \pm 1\}$, and $W_{3} \cong\{ \pm 1\}$. Under these isomorphisms the map $\rho_{W}$ sends $-1 \in\{ \pm 1\}$ to the negative of the identity matrix in $\mathrm{GL}(3, \mathbf{Z})$. 
Proof. The group $\Sigma_{3} l\{ \pm 1\}$ acts on $\bar{L}$ by the formula

$$
\begin{gathered}
\sigma \cdot\left[\varepsilon_{1}, \varepsilon_{2}, \varepsilon_{3}\right] \cdot\left(a_{1} \bar{\ell}_{1}+a_{2} \bar{\ell}_{2}+a_{3} \bar{\ell}_{3}\right)=\varepsilon_{1} a_{1} \bar{\ell}_{\sigma(1)}+\varepsilon_{2} a_{2} \bar{\ell}_{\sigma(2)}+\varepsilon_{3} a_{3} \bar{\ell}_{\sigma(3)} \\
=\varepsilon_{\sigma^{-1}(1)} a_{\sigma^{-1}(1)} \bar{\ell}_{1}+\varepsilon_{\sigma^{-1}(2)} a_{\sigma^{-1}(2)} \bar{\ell}_{2}+\varepsilon_{\sigma^{-1}(3)} a_{\sigma^{-1}(3)} \bar{\ell}_{3}
\end{gathered}
$$

where $\sigma \in \Sigma_{3}, \varepsilon_{i}= \pm 1$ and $a_{i} \in \mathbf{Z}$. Under the isomorphism $\pi_{1} \bar{T} \cong \bar{L}$ this is the conjugation action of the Weyl group of $\mathrm{SO}(7)$ on $\bar{L}$. The action restricts to an action of $W_{1} \cong \Sigma_{3},\{ \pm 1\}$ on the sublattice $L$ of $\bar{L}$, and the stated formula for $\varphi\left(W_{1}\right)$ can be derived by a straightforward calculation. The rest of the lemma follows from the way in which the groups involved are defined. For example, $W_{1}$ acts on $E_{3}$ by conjugation and it is clear that $W_{2}$ is the subgroup of $W_{1}$ consisting of elements which pointwise fix the first two basis elements of $E_{3}$. Since $W_{1}$ acts on $E_{3} \cong L / 2 L$ as $P(1,2)$, the desired identification of $W_{2}$ follows immediately.

3.8. Cohomology of centralizers. To conclude this section we will give a description (3.13) of the cohomology rings of the spaces $B \mathscr{C}\left(E_{i}\right)$ which is functorial enough for the purposes of $\S 6$. All of these cohomology rings are rings of invariants (3.11).

3.9. Lemma (Lannes). Let $G$ be a compact Lie group, $E \subset G$ an elementary abelian 2-subgroup and $W^{\prime}$ the quotient $\mathcal{N}(E) / \mathscr{C}(E)$. Suppose that the natural restriction map $H^{*} B G \rightarrow\left(H^{*} B E\right)^{W^{\prime}}$ is an isomorphism. Let $V$ be an elementary abelian 2-group. Then in the natural commutative square

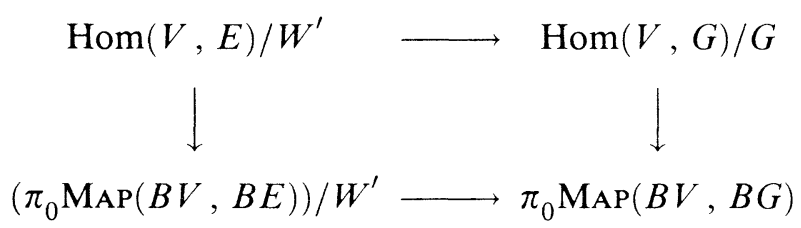

all of the arrows are bijections. Moreover if $h: B V \rightarrow B G$ is a homotopy class of maps, $k: B V \rightarrow B E$ is a homotopy class covering $h$, and $W^{\prime}(k) \subset W^{\prime}$ is the subgroup of elements $w$ such that $w \cdot k=k$, then the evident restriction map $H^{*} \operatorname{MAP}(B V, B G)_{h} \rightarrow\left(H^{*} \operatorname{MAP}(B V, B E)_{k}\right)^{W^{\prime}(k)}$ is an isomorphism.

Remark. Here $\operatorname{Hom}(V, E) / W^{\prime}$ denotes the collection of orbits of the action of $W^{\prime}$ on the set $\operatorname{Hom}(V, E)$, and $\operatorname{Hom}(V, G) / G$ is the set of conjugacy classes of homomorphisms $V \rightarrow G$. Note that for any $k: B V \rightarrow B E$ evaluation at the basepoint gives an equivalence $\operatorname{MAP}(B V, B E)_{k} \rightarrow B E$ which is equivariant with respect to $W^{\prime}(k)$; this equivalence induces the isomorphism $\left(H^{*} \operatorname{MAP}(B V, B E)_{k}\right)^{W^{\prime}(k)} \cong\left(H^{*} B E\right)^{W^{\prime}(k)}$.

Proof of 3.9. This follows from 1.6 and the fact $[20,14]$ that for any compact Lie group $G$ and elementary abelian 2-group $V$ the map

$$
T^{V} H^{*}(B G) \rightarrow H^{*} \operatorname{MAP}(B V, B E)
$$

is an isomorphism. 
For $i=1, \ldots, 4$ let $W_{i}^{\prime}$ denote the quotient group $\mathscr{N}_{i}\left(E_{4}\right) / E_{4}$, where $\mathscr{N}_{i}\left(E_{4}\right)$ is the normalizer of $E_{4}$ in $\mathscr{C}\left(E_{i}\right)$. Choose a basis for $E_{4}$ which extends the basis given above for $E_{3}$, so that the conjugation action of $W_{i}^{\prime}$ on $E_{4}$ gives a homomorphism $W_{i}^{\prime} \rightarrow \mathrm{GL}\left(4, \mathbf{F}_{2}\right)$. Let $P_{0}(i, j)$ denote the kernel of the map $r_{i}: P(i, j) \rightarrow \mathrm{GL}\left(i, \mathbf{F}_{2}\right)$.

3.10. Lemma. The conjugation action of $W_{i}^{\prime}$ on $E_{4}(i=1, \ldots, 4)$ induces an isomorphism $W_{i}^{\prime} \cong P_{0}(i, 4-i)$.

Proof. The map $W_{i}^{\prime} \rightarrow \mathrm{GL}\left(4, \mathbf{F}_{2}\right)$ is clearly a monomorphism; it has image contained in $P_{0}(i, 4-i)$ because the first $i$ basis vectors of $E_{4}$ belong to the center of $\mathscr{C}\left(E_{i}\right)$. Case 1 is proved by observing $(3.4)$ that $W_{1}^{\prime}$ and $P_{0}(1,3)$ have the same order. The rest follows from the fact that essentially by definition $W_{i}^{\prime}(2 \leq i \leq 4)$ is the group of elements in $W_{1}^{\prime}$ which fix the first $i$ basis vectors of $E_{4}$.

3.11. Lemma. For $i=1, \ldots, 4$ the restriction map $H^{*} B \mathscr{C}\left(E_{i}\right) \rightarrow H^{*} B E_{4}$ induces an isomorphism $H^{*} B \mathscr{C}\left(E_{i}\right) \cong\left(H^{*} B E_{4}\right)^{W_{i}^{\prime}}$.

Proof. We will first treat the case $i=1$. According to [2] or [28] the algebra $H^{*} B \operatorname{Spin}(7)$ is a polynomial algebra on classes $x_{4}, x_{6}, x_{7}, x_{8}$. The restriction map $H^{*} B \operatorname{Spin}(7) \rightarrow H^{*} B E_{4}$ makes $H^{*} B E_{4}$ a finitely generated module over $H^{*} B \operatorname{Spin}(7)[29,2.4]$ and, since $H^{*} B E_{4}$ is itself a polynomial algebra on four (one-dimensional) generators, it follows from a transcendence degree argument $[1,11.21]$ that this restriction map must be injective. The ring $H^{*} B E_{4}$ is integral [1, 5.1] over $H^{*} B \operatorname{Spin}(7)$ (this is an easy consequence of the fact that $H^{*} B \operatorname{Spin}(7)$ is Noetherian and $H^{*} B E_{4}$ is finitely generated as a module over $\left.H^{*} B \operatorname{Spin}(7)\right)$. Since the product of the degrees of the polynomial generators of $H^{*} B \operatorname{Spin}(7)$ equals the order of $W_{1}^{\prime}$, the desired isomorphism follows from $[32,3.2]$.

Cases $i=2,3,4$ now follow from 3.9 , since $B \mathscr{C}\left(E_{i}\right)$ is naturally equivalent [14] at the prime 2 to $\operatorname{MAP}\left(B E_{i}, B \operatorname{Spin}(7)\right)_{f}$, where the map $f: B E_{i} \rightarrow$ $B \operatorname{Spin}(7)$ is induced by the inclusion $E_{i} \rightarrow \operatorname{Spin}(7)$.

Let $\delta_{i}: B E_{i} \rightarrow B \mathscr{C}\left(E_{i}\right)$ be induced by the inclusion $E_{i} \rightarrow \mathscr{C}\left(E_{i}\right)$.

3.12. Lemma. Let $V$ be a rank 4 elementary abelian 2-group and let $h: E_{i} \rightarrow$ $V$ be a monomorphism $(i \in\{1, \ldots, 4\})$. Then up to homotopy there is a unique map $k \in \operatorname{EMB}\left(B V, B \mathscr{C}\left(E_{i}\right)\right)$ such that $k \cdot B h$ is homotopic to $\delta_{i}$.

Proof. This is an immediate consequence of $3.11,3.9$ and 3.10 , since up to the action of $W_{i}^{\prime}$ there is only one monomorphism $V \rightarrow E_{4}$ which when composed with $h$ gives the inclusion $E_{i} \rightarrow E_{4}$. Note that by $1.6(2)$ the bijection (3.9) $\operatorname{Hom}\left(V, E_{4}\right) / W_{i}^{\prime} \cong \pi_{0} \operatorname{MAP}\left(B V, B \mathscr{C}\left(E_{i}\right)\right)$ restricts to a bijection $\operatorname{Emb}\left(V, E_{4}\right) / W_{i}^{\prime} \cong \pi_{0} \operatorname{EMB}\left(B V, B \mathscr{C}\left(E_{i}\right)\right)$.

Now let $V$ be a rank 4 elementary abelian 2-group and choose $i \in\{1, \ldots, 4\}$. Lemma 3.12 shows that up to homotopy there is a unique map

$$
\operatorname{EMB}\left(B E_{i}, B V\right) \cong \operatorname{Emb}\left(E_{i}, V\right) \times B V \stackrel{\kappa_{i, v}}{\rightarrow} B \mathscr{C}\left(E_{i}\right)
$$


with the property that for each $f \in \operatorname{EMB}\left(B E_{i}, B V\right)$ the diagram

$$
\begin{array}{cc}
B E_{i} \cong \operatorname{AUT}\left(E_{i}\right)_{1} \stackrel{\delta_{i}}{\longrightarrow} B \mathscr{C}\left(E_{i}\right) \\
f \cdot(-) \downarrow \\
\operatorname{EMB}\left(B E_{i}, B V\right) \stackrel{\kappa_{i, v}}{\longrightarrow} B \mathscr{C}\left(E_{i}\right)
\end{array}
$$

homotopy commutes and such that $\kappa_{i, V}^{*}$ makes $H^{*}$ Емв $\left(B E_{i}, B V\right)$ a finitely generated module over $H^{*} B \mathscr{C}\left(E_{i}\right)$. (The equivalence $\operatorname{Aut}\left(E_{i}\right)_{1} \cong B E_{i}$ in the above diagram is obtained by evaluation at the basepoint $B E_{i}$, as is each equivalence $\operatorname{EMB}\left(B E_{i}, B V\right) \cong B V$.) By uniqueness the map $\kappa_{i, V}$ is equivariant with respect to the evident action of $\operatorname{Aut}(V)$ on its domain and the trivial action of $\operatorname{Aut}(V)$ on its range. It follows that the cohomology map $\kappa_{i, V}^{*}$ induces map from $H^{*} B \mathscr{C}\left(E_{i}\right)$ to be fixed point set of the action of $\operatorname{Aut}(V)$ on $H^{*} \operatorname{EMB}\left(B E_{i}, B V\right)$.

3.13. Proposition. Let $V$ be a rank 4 elementary abelian 2-group. Then for each $i \in\{1, \ldots, 4\}$ the map

$$
H^{*} B \mathscr{C}\left(E_{i}\right) \rightarrow\left(H^{*} \operatorname{EMB}\left(B E_{i}, B V\right)\right)^{\operatorname{Aut}(V)}
$$

induced by $\kappa_{i, v}^{*}$ is an isomorphism.

Proof. Let $h: E_{i} \rightarrow V$ be a monomorphism and $k: B V \rightarrow B \mathscr{C}\left(E_{i}\right)$ the extension of 3.12. Let $W^{\prime} \subset \operatorname{Aut}(V)$ be the subgroup consisting of elements $w$ such that $k \cdot B w$ is homotopic to $k$. By Shapiro's lemma the desired statement amounts to a claim that the map $H^{*} B \mathscr{C}\left(E_{i}\right) \rightarrow\left(H^{*} B V\right)^{W^{\prime}}$ is an isomorphism. This follows from 3.10 and 3.11.

\section{A 2-ADIC REPRESENTATION}

In this section we will show that the conjugation action of the Weyl group of $\operatorname{Spin}(7)$ on the fundamental group of the torus actually extends (after tensoring with $\widehat{\mathbf{Z}}_{2}$ ) to an action of a larger group. This will allow us in $\S 6$ to construct an action of $\operatorname{GL}\left(3, \mathbf{F}_{2}\right)$ on the space $\widehat{B} \mathscr{C}\left(E_{3}\right)$ (see 3.4$)$.

Under the isomorphism $W_{1} \cong P(1,2) \times\{ \pm 1\}$ provided by 3.7 , the homomorphism $\rho_{W}$ gives a map $\rho_{P}: P(1,2) \rightarrow \operatorname{GL}(3, \mathbf{Z})$. Let $\hat{\rho}_{P}$ denote the composite of $\rho_{P}$ with the natural inclusion $\operatorname{GL}(3, \mathbf{Z}) \rightarrow \operatorname{GL}\left(3, \widehat{\mathbf{Z}}_{2}\right)$ and $\hat{\mu}: \operatorname{GL}\left(3, \widehat{\mathbf{Z}}_{2}\right) \rightarrow \mathrm{GL}\left(3, \mathbf{F}_{2}\right)$ the map given by reduction $\bmod 2$.

4.1. Theorem. There is a homomorphism $\hat{\rho}_{\mathrm{GL}}: \mathrm{GL}\left(3, \mathbf{F}_{2}\right) \rightarrow \mathrm{GL}\left(3, \widehat{\mathbf{Z}}_{2}\right)$ such that

(1) the composite $\hat{\mu} \cdot \hat{\rho}_{\mathrm{GL}}$ is the identity map of $\mathrm{GL}\left(3, \mathbf{F}_{2}\right)$, and

(2) the restriction of $\hat{\rho}_{\mathrm{GL}}$ to $P(1,2)$ is $\hat{\rho}_{P}$.

4.2. Remark. By construction the composition $\hat{\mu} \cdot \hat{\rho}_{P}$ gives the identity map of $P(1,2)$. Theorem 4.1 leads immediately to a homomorphism $\operatorname{GL}\left(3, \mathbf{F}_{2}\right) \times$ $\{ \pm 1\} \rightarrow \operatorname{GL}\left(3, \widehat{\mathbf{Z}}_{2}\right)$ which in an evident sense (3.7) extends $\rho_{W}$. 
Let $\operatorname{sl}\left(3, \mathbf{F}_{2}\right)$ denote the additive group of trace zero $3 \times 3$ matrices over $\mathbf{F}_{2}$. The group $\operatorname{GL}\left(3, \mathbf{F}_{2}\right)$ acts on $\operatorname{sl}\left(3, \mathbf{F}_{2}\right)$ by conjugation.

4.3. Lemma. For any subgroup $K$ of $\mathrm{GL}\left(3, \mathbf{F}_{2}\right)$ and $i \geq 1$ the cohomology group $H^{i}\left(K, \operatorname{sl}\left(3, \mathbf{F}_{2}\right)\right)$ vanishes.

Proof. Let $U \subset \mathrm{GL}\left(3, \mathbf{F}_{2}\right)$ be the group of upper-triangular matrices; the group $U$ has order 8 and is a 2-Sylow subgroup of $\operatorname{GL}\left(3, \mathbf{F}_{2}\right)$. A calculation shows that the vector space $H_{0}\left(U, \operatorname{sl}\left(3, \mathbf{F}_{2}\right)\right)$ is of dimension one. Let $x \in \operatorname{sl}\left(3, \mathbf{F}_{2}\right)$ be an element which projects to a generator of $H_{0}\left(U, \operatorname{sl}\left(3, \mathbf{F}_{2}\right)\right)$ and let $f: \mathbf{F}_{2}[U] \rightarrow \operatorname{sl}\left(3, \mathbf{F}_{2}\right)$ be the $\mathbf{F}_{2}[U]$ module map which sends 1 to $x$. Since the augmentation ideal of $\mathbf{F}_{2}[U]$ is nilpotent [4, VI, 8.3] Nakayama's lemma [4, VI, 8.4] shows that the map $f$ is surjective. By a dimension count, then, $f$ is an isomorphism. Now let $K \subset \mathrm{GL}\left(3, \mathbf{F}_{2}\right)$ be any subgroup and $K_{2} \subset K$ its 2-Sylow subgroup; note that $K_{2}$ is conjugate in $\operatorname{GL}\left(3, \mathbf{F}_{2}\right)$ to a subgroup of $U$. It is clear that $\operatorname{sl}\left(3, \mathbf{F}_{2}\right)$ is free as a module over $\mathbf{F}_{2}\left[K_{2}\right]$ and hence [4, VI, 8.5] that the group $H^{i}\left(K_{2}, \operatorname{sl}\left(3, \mathbf{F}_{2}\right)\right)$ vanishes for $i \geq 1$. The lemma follows from a transfer argument [4, III, 10.3].

4.4. Lemma. Suppose that $l: K \rightarrow \mathrm{GL}\left(3, \mathbf{F}_{2}\right)=\mathrm{SL}\left(3, \mathbf{F}_{2}\right)$ is a subgroup inclusion. Then and

(1) there exists a group homomorphism $\rho: K \rightarrow \mathrm{SL}\left(3, \widehat{\mathbf{Z}}_{2}\right)$ such that $\hat{\mu} \cdot \rho=l$,

(2) if $\rho^{\prime}$ is another group homomorphism $K \rightarrow \operatorname{SL}\left(3, \widehat{\mathbf{Z}}_{2}\right)$ with $\hat{\mu} \cdot \rho=l$, then there exists an element $C \in \operatorname{SL}\left(3, \widehat{\mathbf{Z}}_{2}\right)$ such that $\hat{\mu}(C)$ is the identity matrix and $C \rho C^{-1}=\rho^{\prime}$.

Proof. Let $G$ denote $\operatorname{SL}\left(3, \widehat{\mathbf{Z}}_{2}\right)$ and let $G_{n} \subset G(n \geq 1)$ be the normal subgroup consisting of matrices which are congruent to the identity matrix modulo $2^{n}$. It is easy to see that for each $n \geq 1$ the quotient group $G_{n} / G_{n+1}$ is abelian and is isomorphic as a module over $G / G_{n}$ to $\operatorname{sl}\left(3, \mathbf{F}_{2}\right)$, where the action of $G / G_{n}$ on $\operatorname{sl}\left(3, \mathbf{F}_{2}\right)$ is obtained by composing the quotient map $G / G_{n} \rightarrow \mathrm{GL}\left(3, \mathbf{F}_{2}\right)$ with the conjugation action that appears in 4.3.

Suppose that $l$ has been lifted to a homomorphism $\rho_{n}: K \rightarrow G / G_{n}$ for some $n$, and let $q: K^{\prime} \rightarrow K$ be the pullback over $\rho_{n}$ of the surjection $G / G_{n+1} \rightarrow$ $G / G_{n}$. The kernel of $q$ is an abelian group which is isomorphic as a $K$ module to $\operatorname{sl}\left(3, \mathbf{F}_{2}\right)$. By 4.3 the group $H^{2}\left(K, \operatorname{sl}\left(3, \mathbf{F}_{2}\right)\right)$ vanishes and so [4, IV, 3.12] the epimorphism $q$ can be split; moreover, since $H^{1}\left(K, \operatorname{sl}\left(3, \mathbf{F}_{2}\right)\right)$ also vanishes, any two such splittings are conjugate by an element in the kernel of $q[4, \mathrm{IV}, 2.3]$. It follows that the map $\rho_{n}$ lifts to a homomorphism $\rho_{n+1}: K \rightarrow G / G_{n+1}$ and that, if $\rho_{n+1}^{\prime}$ is another such lift, the maps $\rho_{n+1}$ and $\rho_{n+1}^{\prime}$ are conjugate by an element in the kernel of $G / G_{n+1} \rightarrow G / G_{n}$.

By induction, then, the map $l$ lifts for any $n$ to a homomorphism $\rho_{n}: K \rightarrow$ $G / G_{n}$, and any two such lifts are conjugate via an element $C_{n} \in G / G_{n}$ which projects to the identity element of $\operatorname{GL}\left(3, \mathbf{F}_{2}\right)$. Let $\mathscr{L}(n)$ be the set $\left\{\rho_{n}\right\}$ of all such lifts. There are natural surjections $\mathscr{L}(n) \rightarrow \mathscr{L}(n-1)$, and $\mathscr{L}(\infty)=$ $\lim \mathscr{L}(n)$ is the set of all homomorphisms $\rho: K \rightarrow G$ such that $\hat{\mu} \cdot \rho=\imath$. It is clear that $\mathscr{L}(\infty)$ is nonempty. This proves (1). 
Now suppose that $\rho$ and $\rho^{\prime}$ are elements of $\mathscr{L}(\infty)$. Let $\rho_{n}$ and $\rho_{n}^{\prime}$ denote the images of these homomorphisms in $\mathscr{L}(n)$, and let $\mathscr{C}(n)$ denote the set of all elements of $G / G_{n}$ which conjugate $\rho_{n}$ to $\rho_{n}^{\prime}$ and project trivially to $\mathrm{GL}\left(3, \mathbf{F}_{2}\right)$. There are natural maps $\mathscr{C}(n) \rightarrow \mathscr{C}(n-1)$, and $\mathscr{C}(\infty)=\lim \mathscr{C}(n)$ is the set of all elements of $G$ which conjugate $\rho$ to $\rho^{\prime}$ and project trivially to $\mathrm{GL}\left(3, \mathbf{F}_{2}\right)$. Part (2) of the lemma follows from the fact that $\mathscr{C}(\infty)$ is nonempty, since each $\mathscr{C}(n)$ is finite and nonempty.

Proof of 4.1. By part (1) of 4.4 applied to $K=\mathrm{GL}\left(3, \mathbf{F}_{2}\right)$, there exists a homomorphism $\hat{\rho}_{\mathrm{GL}}: \operatorname{GL}\left(3, \mathbf{F}_{2}\right) \rightarrow \mathrm{GL}\left(3, \widehat{\mathbf{Z}}_{2}\right)$ which satisfies (1) of 4.1. By part (2) of 4.4 applied to $K=P(1,2)$ (see 4.2$)$, the restriction of $\hat{\rho}_{\mathrm{GL}}$ to $P(1,2)$ is conjugate to $\hat{\rho}_{P}$ via an element $C \in \mathrm{GL}\left(3, \widehat{\mathbf{Z}}_{2}\right)$ which projects trivially to $\mathrm{GL}\left(3, \mathbf{F}_{2}\right)$. The proof is completed by replacing $\hat{\rho}_{\mathrm{GL}}$ with its conjugate by $C$.

\section{SElF-MAPS OF COMPLETED CLASSifying SPACES}

In this section we will calculate (5.5) the space of self homotopy equivalences of $\widehat{B} Q_{\text {red }}^{n}$ (see 3.4). This will allow us in $\S 6$ to construct an action of $\operatorname{GL}\left(2, \mathbf{F}_{2}\right)$ on the space $\widehat{B} \mathscr{C}\left(E_{2}\right) \cong \widehat{B} Q_{\text {red }}^{3}$. There is an evident permutation action of $\mathrm{GL}\left(2, \mathbf{F}_{2}\right) \cong \Sigma_{3}$ on $\widehat{B} Q_{\text {red }}^{3}$, but the action which comes up in $\S 6$ is another one.

Some of what we do is implicit in $[14,10,27]$, although the particular results we need do not appear in these references. For the rest of the section let $G$ denote a compact Lie group with chosen maximal torus $T$ and Weyl group $W$.

The set $\operatorname{Emb}(T, G)$ (see 1.9) has a distinguished basepoint given by the inclusion of $T$ i n $G$. Since $T$ is unique up to conjugacy the discrete group $\operatorname{Aut}(T)$ acts transitively by composition on $\operatorname{Emb}(T, G)$; it is clear that the isotropy group of the distinguished basepoint is the image of the map $W \rightarrow \operatorname{Aut}(T)$ given by the conjugation action of $W$ on $T$. We will need a homotopy-theoretic version of this fact. Note that the set $\pi_{0} \operatorname{EMB}(\widehat{B} T, \widehat{B} G)$ has a distinguished basepoint given by the map $\widehat{B} T \rightarrow \widehat{B} G$ induced by the inclusion $T \rightarrow G$, and that the discrete group $\pi_{0} \operatorname{AUT}(\widehat{B} T)$ acts on $\pi_{0} \operatorname{EMB}(\widehat{B} T, \widehat{B} G)$ by composition.

5.1. Proposition. If $G$ is a connected compact Lie group then the natural action of $\pi_{0} \operatorname{AuT}(\widehat{B} T)$ on $\pi_{0} \operatorname{EMB}(\widehat{B} T, \widehat{B} G)$ is transitive; the isotropy group of the distinguished basepoint is the image of the map $W \rightarrow \pi_{0} \operatorname{AUT}(\widehat{B} T)$ given by the conjugation action of $W$ on $T$.

5.2. Remark. This will be proven below. Note that choice of a basis for $\pi_{1} T$ gives isomorphisms $\operatorname{Aut}(T) \cong \mathrm{GL}(r, \mathbf{Z})$ and $\pi_{0} \operatorname{AuT}(\widehat{B} T) \cong \mathrm{GL}\left(r, \widehat{\mathbf{Z}}_{2}\right)$, where $r$ is the rank of $G$.

5.3. Lemma. Suppose that $G$ is connected and that $\pi$ is a finite two-group. Then

(1) the natural map $\operatorname{MAP}(B \pi, B G) \rightarrow \operatorname{MAP}(B \pi, \widehat{B} G)$ induces an isomorphism on mod 2 cohomology, and

(2) the space $\operatorname{MAP}(B \pi, \widehat{B} G)$ is $\mathbf{F}_{2}$-complete in the sense of [3]. 
Remark. Lemma 5.3 implies that $\operatorname{MAP}(B \pi, \widehat{B} G)$ is homotopy equivalent to the $\mathbf{F}_{2}$-completion of $\operatorname{MAP}(B \pi, B G)$.

Proof of 5.3. Part (1) is proved by the argument of $[6,4.5]$; the main step is to note that the homotopy fibre of the map $B G \rightarrow \widehat{B} G$ is a simple space with uniquely 2-divisible homotopy groups. Part (2) now follows from [3, Chapter VII, 5.1] and the fact $[14,1.1]$ that each component of $\operatorname{MAP}(B \pi, B G)$ has a finite fundamental group.

Proof of 5.1. Let $T_{n}$ be the subgroup of $T$ consisting of elements $x$ such that $x^{2^{n}}=1$, and let $T_{\infty}$ denote the discrete group $\cup_{n} T_{n}$. The composite map $B T_{\infty} \rightarrow B T \rightarrow \widehat{B} T$ is a cohomology isomorphism and so induces an isomorphism $\pi_{0} \operatorname{EMB}(\widehat{B} T, \widehat{B} G) \cong \pi_{0} \operatorname{EMB}\left(B T_{\infty}, \widehat{B} G\right)$. It is easy to see by explicit calculation that the functorial map $\operatorname{Aut}\left(T_{\infty}\right) \rightarrow \pi_{0} \operatorname{Aut}\left(\widehat{B} T_{\infty}\right) \cong \pi_{0} \operatorname{AuT}(\widehat{B} T)$ is an isomorphism. To prove 5.1, then, it is enough to show that $\operatorname{Aut}\left(T_{\infty}\right)$ acts transitively on $\pi_{0} \operatorname{EMB}\left(B T_{\infty}, \widehat{B} G\right)$ and that the isotropy group of the evident distinguished basepoint is the image of the map $W \rightarrow \operatorname{Aut}\left(T_{\infty}\right)$ given by the conjugation action of $W$ on $T_{\infty}$.

Since $B T_{\infty}$ is equivalent to the homotopy direct limit holim $B T_{n}$ [3, XII, 3.6], the mapping space $\operatorname{EMB}\left(B T_{\infty}, \widehat{B} G\right)$ is equivalent to the homotopy inverse limit $\operatorname{holim} \operatorname{EmB}\left(B T_{n}, \widehat{B} G\right) \quad\left(\right.$ see [3, XII, 4.1]). Choose $n_{0} \gg 0$ so that for $n>n_{0}$

(1) the map $\operatorname{Emb}\left(T_{n+1}, T\right) \rightarrow \operatorname{Emb}\left(T_{n}, G\right)$ is surjective, and

(2) the centralizer of $T_{n}$ in $G$ is exactly $T$.

(It is easy to obtain (1) for large $n$ by observing that any $f: T_{n} \rightarrow G$ has image contained up to conjugacy in the normalizer $\mathscr{N}(T)$ of $T^{n}$ [30] and then using the fact that $W=T / \mathscr{N}(T)$ is a finite group. For (2), let $C_{n}$ be the centralizer in $G$ of $T_{n}$, observe that $T$ is self-centralizing and $T_{\infty}$ is dense in $T$ so that $\bigcap_{n} C_{n}=T$, and use a dimension argument to conclude that $C_{n}=T$ for sufficiently large $n$.) By $[14,1.1]$ and 5.3 the natural map $\operatorname{Emb}\left(T_{n}, G\right) \rightarrow \pi_{0} \operatorname{EMB}\left(B T_{n}, \widehat{B} G\right)$ is an isomorphism for any $n$ and for $n>n_{0}$ each component of $\operatorname{EMB}\left(B T_{n}, \widehat{B} G\right)$ is naturally homotopy equivalent to $\widehat{B} T$. It follows [3, IX, 3.5] that $\lim ^{1} \pi_{1} \operatorname{EMB}\left(B T_{n}, \widehat{B} G\right)$ vanishes for every choice of a compatible sequence of basepoints and [3, XI, 4.1; IX, 3.1] that there is an isomorphism $\pi_{0} \operatorname{EMB}\left(B T_{\infty}, \widehat{B} G\right) \cong \lim \operatorname{Emb}\left(T_{n}, G\right)$. The desired result can now be proven by combining the isomorphism $\operatorname{Aut}\left(T_{\infty}\right) \cong \lim \operatorname{Aut}\left(T_{n}\right)$ with the fact that for $n>n_{0}$ the action of $\operatorname{Aut}\left(T_{n}\right)$ on $\operatorname{Emb}\left(T_{n}, \overleftarrow{G)}\right.$ is clearly transitive and has the conjugation image of $W$ in $\operatorname{Aut}\left(T_{n}\right)$ as the isotropy group of the natural basepoint.

Now suppose that $G$ is connected. Since $T$ is self-centralizing the conjugation action of $W$ on $\widehat{B} T$ is faithful, and we will use this action to identify $W$ with a subgroup of $\pi_{0} \operatorname{AUT}(\widehat{B} T)$. Let $\nu=\nu_{G}$ denote the normalizer of $W$ in $\pi_{0} \operatorname{Aut}(\widehat{B} T)$ and $\bar{\nu}=\bar{\nu}_{G}$ the quotient of $\nu$ by $W$. By 5.1, $\bar{\nu}$ is isomorphic to the group of automorphisms of $\pi_{0} \operatorname{EMB}(\widehat{B} T, \widehat{B} G)$ as a set with an 
action of $\pi_{0} \operatorname{AUT}(\widehat{B} T)$. Under this isomorphism, the left composition action of $\pi_{0} \operatorname{Aut}(\widehat{B} G)$ on $\pi_{0} \operatorname{EmB}(\widehat{B} T, \widehat{B} G)$ gives a map $\chi=\chi_{G}: \pi_{0} \operatorname{Aut}(\widehat{B} G) \rightarrow \bar{\nu}$.

Recall that $\mathscr{Z}(G)$ denotes the center of the compact Lie group $G$. As in $[14, \S 1]$ the group homomorphism $\mathscr{Z}(G) \times G \rightarrow G$ gives a map $B \mathscr{Z}(G) \rightarrow$ $\operatorname{Aut}(\widehat{B} G)_{1}$.

5.4. Lemma. The space $\operatorname{MAP}(\widehat{B} Q, \widehat{B} Q)$ is the union of $\operatorname{Aut}(\widehat{B} Q)$ with the component $\operatorname{MAP}(\widehat{B} Q, \widehat{B} Q)_{0}$ of null homotopic maps. Moreover

(1) the map $\chi_{Q}: \pi_{0} \operatorname{AUT}(\widehat{B} Q) \rightarrow \bar{\nu}_{Q}$ is an isomorphism,

(2) the natural map $B \mathscr{Z}(Q) \rightarrow \operatorname{AUT}(\widehat{B} Q)_{1}$ is an equivalence, and

(3) the basepoint evaluation map $\operatorname{MAP}(\widehat{B} Q, \widehat{B} Q)_{0} \rightarrow \widehat{B} Q$ is an equivalence.

Remark. The group $\mathscr{Z}(Q)$ is $\{ \pm 1\}$ and the group $\bar{\nu}_{Q}$ is canonically isomorphic to $\widehat{\mathbf{Z}}_{2}^{\times} /\{ \pm 1\}$.

Proof. Let $\widetilde{\mathscr{O}}_{48}$ and $Q_{16}$ be the subgroups of $Q$ constructed in $[8, \S 4]$ and let $\mathscr{N}_{2} T \subset Q$ be the subgroup of the normalizer of the torus in $Q$ generated by $T_{\infty}$ and some $X \notin T$. It follows from [8, 4.1] that there is a diagram $B \widetilde{\mathscr{O}}_{48} \leftarrow B Q_{16} \rightarrow B \mathscr{N}_{2} T$ induced by subgroup inclusions and a map from the homotopy pushout $P$ of this diagram to $\widehat{B} Q$ which induces an isomorphism on cohomology. As a consequence the space $\operatorname{MAP}(\widehat{B} Q, \widehat{B} Q) \cong \operatorname{MAP}(P, \widehat{B} Q)$ is equivalent to the homotopy pullback of the induced diagram

$$
\operatorname{MAP}\left(B \widetilde{\mathscr{O}}_{48}, \widehat{B} Q\right) \rightarrow \operatorname{MAP}\left(B Q_{16}, \widehat{B} Q\right) \leftarrow \operatorname{MAP}\left(B \mathscr{N}_{2} T, \widehat{B} Q\right) .
$$

By $[14,1.1], 5.3$, and a limiting argument based on the fact that $\mathscr{N}_{2} T$ is a union of finite two-groups (cf. proof of 5.1), it is possible to identify $\operatorname{MAP}\left(B \mathscr{N}_{2} T, \widehat{B} Q\right)$ up to homotopy as a disjoint union involving one copy of $\widehat{B} Q$ corresponding to the component of null homotopic maps together with multiple copies of $B\{ \pm 1\}$ (one for each element $\bar{\nu}_{Q}$ ). Similarly, the subspace of $\operatorname{MAP}\left(B Q_{16}, \widehat{B} Q\right)$ consisting of maps which extend up to homotopy to $B \mathscr{N}_{2} T$ is up to homotopy the disjoint union of $\widehat{B} Q$ with two copies of $B\{ \pm 1\}$. Finally, both $Q_{16}$ and $\widetilde{\mathscr{O}}_{48}$ contain a normal subgroup $Q_{8}$ of order 8 (essentially the classical quaternion group); the subspace $M_{e}$ of $M=\operatorname{MAP}\left(B Q_{8}, \widehat{B} Q\right)$ consisting of maps which extend up to homotopy to $B \mathscr{N}_{2} T$ is up to homotopy the disjoint union of $\widehat{B} Q$ with one copy of $B\{ \pm 1\}$. As in $[14,5.1]$ there are equivalences

$$
\begin{aligned}
& \operatorname{MAP}\left(B \widetilde{\mathscr{O}}_{48}, \widehat{B} Q\right) \cong M^{\mathrm{h} \gamma}, \\
& \operatorname{MAP}\left(B Q_{16}, \widehat{B} Q\right) \cong M^{\mathrm{h} \gamma^{\prime}},
\end{aligned}
$$

where we have used the homotopy fixed point set notation $(-)^{\mathrm{h}-}$ of [14], $\gamma=$ $\widetilde{\mathscr{O}}_{48} / Q_{8}$ and $\gamma^{\prime}=Q_{16} / Q_{8}$. Now $\gamma$ is isomorphic to the symmetric group $\Sigma_{3}$ and so has a normal subgroup $\gamma^{\prime \prime}$ of order 3 with $\gamma / \gamma^{\prime \prime} \cong \gamma^{\prime}$. It is easy to see that $\gamma^{\prime \prime}$ acts trivially up to homotopy on $M_{e}$ and thus by the argument of $[14,2.3]$ that $M_{e}^{\mathrm{h} \gamma^{\prime \prime}} \cong M_{e}$. By the argument of $[14,5.1]$ there is an equivalence $M_{e}^{\mathrm{h} \gamma} \cong$ 
$\left(M_{e}^{\mathrm{h} \gamma^{\prime \prime}}\right)^{\mathrm{h} \gamma^{\prime}}$. It follows that $M_{e}^{\mathrm{h} \gamma} \cong M_{e}^{\mathrm{h} \gamma^{\prime}}$, which implies that the above homotopy pullback collapses to an equivalence $\operatorname{MAP}(\widehat{B} Q, \widehat{B} Q) \cong \operatorname{MAP}\left(B \mathcal{N}_{2} T, \widehat{B} Q\right)$. The desired result follows directly.

5.5. Proposition. Suppose that $G$ is $Q^{n}$ or $Q_{\mathrm{red}}^{n}(n>0)$. Then

(1) the map $\chi_{G}: \pi_{0} \operatorname{AUT}(\widehat{B} G) \rightarrow \bar{\nu}_{G}$ is an isomorphism, and

(2) the natural map $B \mathscr{Z}(G) \rightarrow \operatorname{Aut}(\widehat{B} G)_{1}$ is an equivalence.

Proof. It is clear that evaluation at the basepoint gives an equivalence

$$
\operatorname{MAP}(\widehat{B} Q, B \mathscr{Z}(Q)) \rightarrow B \mathscr{Z}(Q) .
$$

It follows from an inductive argument using 5.4 and the formula

$$
\operatorname{MAP}\left(\widehat{B} Q^{n}, \widehat{B} Q\right) \cong \operatorname{MAP}\left(\widehat{B} Q, \operatorname{MAP}\left(\widehat{B} Q^{n-1}, \widehat{B} Q\right)\right)
$$

that any map $\widehat{B} Q^{n} \rightarrow \widehat{B} Q$ factors up to homotopy through one of the $n$ evident projections and that via these projections $\operatorname{MAP}\left(\widehat{B} Q^{n}, \widehat{B} Q\right)$ is equivalent to an amalgamated union of $n$ copies of $\operatorname{MAP}(\widehat{B} Q, \widehat{B} Q)$ (the amalgamation amounts to identifying the subspaces of null homotopic maps). An argument along the lines of $[16, \S 4]$ now shows that the obvious map $\Sigma_{n} \backslash \operatorname{AUT}(\widehat{B} Q) \rightarrow \operatorname{AUT}\left(\widehat{B} Q^{n}\right)$ is an equivalence. Since $\bar{\nu}_{Q^{n}} \cong \Sigma_{n} i \bar{\nu}_{Q}$ and $\mathscr{Z}\left(Q^{n}\right) \cong \mathscr{Z}(Q)^{n}$ the desired result follows easily in the case $G=Q^{n}$.

For the case $G=Q_{\text {red }}^{n}$, let $K$ denote the Eilenberg-Mac Lane space $K(\mathbf{Z} / 2,2)$ and note that there is a fibration $p: \widehat{B} Q_{\text {red }}^{n} \rightarrow K$ (with fibre $\widehat{B} Q^{n}$ ) which is the projection of $\widehat{B} Q_{\text {red }}^{n}$ to its second Postnikov stage. Since the formulation of Postnikov stages is functorial, the topological monoid of selfequivalences of $p$ in the sense of $[7,6.1]$ is equivalent to $\operatorname{AuT}\left(\widehat{B} Q_{\text {red }}^{n}\right.$ ) (cf. [7, $6.3])$. By $[7,6.2]$, then, there is a natural weak equivalence

$$
B \operatorname{Aut}\left(\widehat{B} Q_{\text {red }}^{n}\right) \cong E \operatorname{Aut}(K) \times_{\operatorname{Aut}(K)} \operatorname{MAP}\left(K, B \operatorname{Aut}\left(\widehat{B} Q^{n}\right)\right)_{p}
$$

(where the subscript $p$ on the right denotes the component of the indicated mapping space which contains a classifying map for $p)$. Since $\operatorname{AUT}(K) \cong$ $K$ and $\operatorname{MAP}\left(K, B \operatorname{AUt}\left(\widehat{B} Q^{n}\right)\right)_{p} \cong B \operatorname{AUt}\left(\widehat{B} Q^{n}\right)$ (both via basepoint evaluation maps), it is not hard to calculate directly that the natural map $\mathscr{Z}\left(Q_{\text {red }}^{n}\right) \rightarrow$ $\pi_{2} B \operatorname{AuT}\left(\widehat{B} Q_{\text {red }}^{n}\right)$ is an isomorphism. It is also clear that every self-equivalence of $\widehat{B} Q_{\text {red }}^{n}$ is covered up to homotopy by a unique self-equivalence of $\widehat{B} Q^{n}$. The proof is completed by checking that if $\widetilde{T}$ is a maximal torus of $Q^{n}$ which covers the maximal torus $T$ of $Q_{\text {red }}^{n}$, then each automorphism of $T$ which normalizes the action of the Weyl group of $Q_{\text {red }}^{n}$ lifts to a unique automorphism of $\tilde{T}$ which normalizes the action of the Weyl group of $Q^{n}$.

\section{A CONSTRUCTION IN THE HOMOTOPY CATEGORY}

Let $\operatorname{Ho}(\mathscr{T})$ be the homotopy category of $\mathscr{T}$. In this section we will define a functor $\bar{F}: \mathbf{A}^{\text {op }} \rightarrow \operatorname{Ho}(\mathscr{T})$ such that $H^{*} \bar{F}$ is naturally equivalent to the functor $\alpha_{R}$ of 2.5. We will continue to use the notation of $\xi 3$. 
By construction each vector space $E_{i}, i=1, \ldots, 4$, comes with a basis and therefore an action of $\operatorname{GL}\left(i, \mathbf{F}_{2}\right)$; we will refer to the induced action of $\mathrm{GL}\left(i, \mathbf{F}_{2}\right)$ on $B E_{i}$ as the basis action of $\mathrm{GL}\left(i, \mathbf{F}_{2}\right)$ on $B E_{i}$. By 3.6 and 3.7 the internal structure of $\operatorname{Spin}(7)$ provides actions up to homotopy of $P(1,1) \cong$ $W_{2}^{+} / W_{2}$ on $\widehat{B} \mathscr{C}\left(E_{2}\right)$ and of $P(1,2) \cong W_{3}^{+} / W_{3}$ on $\widehat{B} \mathscr{C}\left(E_{3}\right)$; we will refer to these as the internal Spin actions of these groups. Let $\hat{\delta}_{i}: B E_{i} \rightarrow \widehat{B} \mathscr{C}\left(E_{i}\right)$ be the map induced by the inclusion $E_{i} \rightarrow \mathscr{C}\left(E_{i}\right)$.

6.1. Proposition. There are homotopy actions of $\mathrm{GL}\left(i, \mathbf{F}_{2}\right)$ on $\widehat{B} \mathscr{C}\left(E_{i}\right), i=$ 2,3 , with the following properties.

(1) The map $\hat{\delta}_{i}: B E_{i} \rightarrow \widehat{B} \mathscr{C}\left(E_{i}\right)$ is equivariant with respect to the basis action of $\mathrm{GL}\left(i, \mathbf{F}_{2}\right)$ on $B E_{i}$ for $i=2,3$.

(2) The restriction to $P(1,2)$ of the action of $\mathrm{GL}\left(3, \mathbf{F}_{2}\right)$ agrees with the internal Spin action of $P(1,2)$ on $\widehat{B} \mathscr{C}\left(E_{3}\right)$.

(3) The restriction to $P(1,1)$ of the action of $\mathrm{GL}\left(2, \mathbf{F}_{2}\right)$ agrees with the internal Spin action of $P(1,1)$ on $\widehat{B} \mathscr{C}\left(E_{2}\right)$.

Restricting the action of $\mathrm{GL}\left(3, \mathbf{F}_{2}\right)$ to $P(2,1)$ gives an action of $P(2,1)$ on $\widehat{B} \mathscr{C}\left(E_{3}\right)$ and composing the action of $\mathrm{GL}\left(2, \mathbf{F}_{2}\right)$ with the natural projection $P(2,1) \rightarrow P(2,1) / U(2,1) \cong \mathrm{GL}\left(2, \mathbf{F}_{2}\right)$ gives an action of $P(2,1)$ on $\widehat{B} \mathscr{C}\left(E_{2}\right)$.

(4) The map $\widehat{B} \mathscr{C}\left(E_{3}\right) \rightarrow \widehat{B} \mathscr{C}\left(E_{2}\right)$ induced by the inclusion $\mathscr{C}\left(E_{3}\right) \rightarrow \mathscr{C}\left(E_{2}\right)$ is equivariant with respect to these actions of $P(2,1)$.

Proof. Let $\chi_{i}: \pi_{0} \operatorname{AuT}\left(\widehat{B} \mathscr{C}\left(E_{i}\right)\right) \rightarrow \bar{\nu}_{i}$ be the map $\chi_{G}$ of $\S 5$ for $G=\mathscr{C}\left(E_{i}\right)$, $i=2,3$. The map $\chi_{2}$ is an isomorphism by 5.5. It is easy to see that $\chi_{3}$ is well-defined (in spite of the fact that $\mathscr{C}\left(E_{3}\right)$ is not connected) and induces an isomorphism from $\pi_{0} \operatorname{AUT}\left(\widehat{B} \mathscr{C}\left(E_{3}\right)\right)$ to the quotient of $\operatorname{GL}\left(3, \widehat{\mathbf{Z}}_{2}\right)$ by the subgroup $\left\{ \pm I_{3}\right\}$ generated by the negative of the identity matrix. (Here and in what follows we use the specific basis $\left\{\ell_{1}, \ell_{2}, \ell_{3}\right\}$ of $L \cong \pi_{2} B T$ to identify $\pi_{0} \operatorname{Aut}(\widehat{B} T)$ with $\mathrm{GL}\left(3, \widehat{\mathbf{Z}}_{2}\right)$.) The representation $\hat{\rho}_{\mathrm{GL}}$ of 4.1 can be composed with the quotient map $\operatorname{GL}\left(3, \widehat{\mathbf{Z}}_{2}\right) \rightarrow \operatorname{GL}\left(3, \widehat{\mathbf{Z}}_{2}\right) /\left\{ \pm I_{3}\right\}$ and the inverse of $\chi_{3}$ to give an action of $\mathrm{GL}\left(3, \mathbf{F}_{2}\right)$ on $\widehat{B} \mathscr{C}\left(E_{3}\right)$. Condition $(1)(i=3)$ follows from the isomorphism $E_{3} \cong L / 2 L$ together with 4.1 (1), condition (2) from 3.7 and $4.1(2)$.

The group $U(2,1)$ is a subgroup of $P(1,2)$. By 3.7 the image of $W_{2}$ in $\operatorname{GL}\left(3, \widehat{\mathbf{Z}}_{2}\right)$ is generated by $\left\{ \pm I_{3}\right\}$ and $(4.1) \hat{\rho}_{P}(U(2,1))$, where by $4.1(2)$ this last group is equal to $\hat{\rho}_{\mathrm{GL}}(U(2,1))$. Since $U(2,1)$ is a normal subgroup of $P(2,1)$, it follows that $\hat{\rho}_{\mathrm{GL}}(P(2,1))$ is contained in the normalizer of the image of $W_{2}$ and hence that $\hat{\rho}_{\mathrm{GL}}$ induces a map $P(2,1) / U(2,1) \cong \mathrm{GL}\left(2, \mathbf{F}_{2}\right) \rightarrow$ $\bar{\nu}_{2} \cong \pi_{0} \operatorname{AUT}\left(\widehat{B} \mathscr{C}\left(E_{2}\right)\right)$. Use this map to give an action of $\operatorname{GL}\left(2, \mathbf{F}_{2}\right)$ on $\widehat{B} \mathscr{C}\left(E_{2}\right)$. Condition (1) $(i=2)$ follows from 4.1(1) and the natural isomorphism between $E_{2}$ and the subgroup of $L / 2 L$ generated by the residue classes of $\ell_{1}$ and $\ell_{2}$.

Restricting the above action of $\operatorname{GL}\left(2, \mathbf{F}_{2}\right)$ on $\widehat{B} \mathscr{C}\left(E_{2}\right)$ to $P(1,1)$ and ap- 
plying $\chi_{2}$ gives a monomorphism

$$
P(1,1)=U / U(2,1) \rightarrow \bar{\nu}_{2}=\mathcal{N}\left(\hat{\rho}_{\mathrm{GL}}(U(2,1))\right) / \hat{\rho}_{\mathrm{GL}}(U(2,1))
$$

(see 3.7) which is covered by the map $U \rightarrow \mathrm{GL}\left(3, \widehat{\mathbf{Z}}_{2}\right)$ given by the restriction to $U$ of $\hat{\rho}_{\mathrm{GL}}$. The internal Spin action of $P(1,1)$ on $\widehat{B} \mathscr{C}\left(E_{2}\right)$ induces a parallel monomorphism $P(1,1) \rightarrow \bar{\nu}_{2}$ which is covered by the map $U \rightarrow$ $\operatorname{GL}\left(3, \widehat{\mathbf{Z}}_{2}\right)$ given by the restriction to $U$ of $\hat{\rho}_{P}(3.7,4.1)$. Condition (3) is thus a consequence of 4.1(2).

Finally, property (4) is clear by construction.

Lemma 2.1 describes what is needed to build $\bar{F}$. Choose $\bar{F}\left(A_{i}\right)=\widehat{B} \mathscr{C}\left(E_{i}\right)$, $i=1, \ldots, 4$. The maps $\bar{F}\left(f_{i}\right) \quad(i=1,2,3)$ are induced by the inclusions $\mathscr{C}\left(E_{i+1}\right) \rightarrow \mathscr{C}\left(E_{i}\right)$. The right actions of $\mathrm{GL}\left(2, \mathbf{F}_{2}\right)$ and $\operatorname{GL}\left(3, \mathbf{F}_{2}\right)$ on $\bar{F}\left(A_{2}\right)$ and $\bar{F}\left(A_{3}\right)$ respectively are obtained by composing the left actions of 6.1 with the inverse map. The right action of $\mathrm{GL}\left(4, \mathbf{F}_{2}\right)$ on $\bar{F}\left(A_{4}\right)$ is obtained in a similar way from the natural left action of $\mathrm{GL}\left(4, \mathbf{F}_{2}\right)$ on the based vector space $E_{4}=\mathscr{C}\left(E_{4}\right)$. By $6.1(3)$ the subgroup $P(1,1) \subset \operatorname{GL}\left(2, \mathbf{F}_{2}\right)$ acts on $\bar{F}\left(A_{2}\right)$ by automorphisms of $\mathscr{C}\left(E_{2}\right)$ which extend to inner automorphisms of $\operatorname{Spin}(7)$; this (cf. 3.6) gives relation 2.1(1). Relation 2.1(2) is 6.1(4). By 6.1(2), the restriction of the action of $\mathrm{GL}\left(3, \mathbf{F}_{2}\right)$ on $\bar{F}\left(A_{3}\right)$ to $P(1,2)$ is induced by automorphisms of $\mathscr{C}\left(E_{3}\right)$ which extend to inner automorphisms of $\operatorname{Spin}(7)$; this establishes relation 2.1(4). Finally, relation 2.1(6) follows from 3.10, since the action of $P(1,3)$ on $\widehat{B} \mathscr{C}\left(E_{4}\right)$ is induced by group automorphisms which become inner in $\operatorname{Spin}(7)$.

Relations 2.1(3) and 2.1(5) require a little more work. For 2.1(3), let $T_{\mathrm{ext}}^{n}(2)$ denote some subgroup of $T_{\text {ext }}^{n}$ (see 3.4) which is isomorphic to the semidirect product of $\left(\mathbf{Z}_{2^{\infty}}\right)^{n}$ with $\mathbf{Z} / 2$. The inclusion $T_{\text {ext }}^{n}(2) \rightarrow T_{\text {ext }}^{n}$ induces a cohomology isomorphism $H^{*} T_{\text {ext }}^{n} \rightarrow H^{*} T_{\text {ext }}^{n}(2)$ and therefore an equivalence $\widehat{B} T_{\text {ext }}^{n}(2) \cong \widehat{B} T_{\text {ext }}^{n}$. Under this equivalence the above action of $\operatorname{GL}\left(3, \mathbf{F}_{2}\right)$ on $\widehat{B} \mathscr{C}\left(E_{3}\right) \cong \widehat{B} T_{\text {ext }}^{3}$ is by construction induced by an action of $\operatorname{GL}\left(3, \mathbf{F}_{2}\right)$ on $T_{\mathrm{ext}}^{n}(2)$ by group homomorphisms. This last action is obtained by letting $\operatorname{GL}\left(3, \mathbf{F}_{2}\right)$ act via $\hat{\rho}_{\mathrm{GL}}$ on $\left(\mathbf{Z}_{2 \infty}\right)^{3}$ (note that $\operatorname{Aut}\left(\left(\mathbf{Z}_{2 \infty}\right)^{3}\right) \cong \mathrm{GL}\left(3, \widehat{\mathbf{Z}}_{2}\right)$ ) and then extending to $T_{\text {ext }}^{3}(2)$ by using the fact that $-I_{3} \in \operatorname{GL}\left(3, \widehat{Z}_{2}\right)$ commutes with $\hat{\rho}_{\mathrm{GL}}\left(\mathrm{GL}\left(3, \mathbf{F}_{2}\right)\right)$. In a similar way, the map $\bar{F}\left(f_{3}\right): \widehat{B} E_{4} \rightarrow \widehat{B} \mathscr{C}\left(E_{3}\right)$ is induced by a group homomorphism $E_{4} \rightarrow T_{\mathrm{ext}}^{3}(2)$ which sends the first three basis vectors of $E_{4}$ to elements of order 2 in the subgroup $\left(\mathbf{Z}_{2^{\infty}}\right)^{3}$ and the last basis vector to an element of order 2 which does not lie in $\left(\mathbf{Z}_{2^{\infty}}\right)^{3}$. Relation 2.1(3) now follows from a straightforward group-theoretic calculation involving $6.1(1)$; note in particular that the automorphisms of $E_{4}$ provided by $U(3,1)$ become inner automorphisms in $T_{\text {ext }}^{3}(2)$ (or in $\mathscr{C}\left(E_{3}\right)$ ).

For 2.1(5) observe (3.10) that the automorphisms of $E_{4}$ provided by $P_{0}(2,2)$ become inner automorphisms in $\mathscr{C}\left(E_{2}\right)$, so it is enough to check 2.1 (5) for matrices $h \in \mathrm{GL}\left(4, \mathbf{F}_{2}\right)$ which agree with the identity except in the upper left-hand $2 \times 2$ block. Such a matrix $h$ belongs to $P(3,1)$, so that by $2.1(3) \bar{F}\left(f_{3}\right) \bar{F}(h)=$ 
$\bar{F}\left(r_{3}(h)\right)$ and hence by $2.1(2) \bar{F}\left(f_{2}\right) \bar{F}\left(f_{3}\right) \bar{F}(h)=\bar{F}\left(r_{2} r_{3}(h)\right) \bar{F}\left(f_{2}\right) \bar{F}\left(f_{3}\right)$. This immediately gives $2.1(5)$ and completes the proof that $\bar{F}$ is a functor $\mathbf{A}^{\text {op }} \rightarrow$ $\operatorname{Ho}(\mathscr{T})$.

Next we show that the functor $H^{*} \bar{F}$ is naturally equivalent to $\alpha_{R}$. Let $E_{\mathbf{A}}: \mathbf{A} \rightarrow \mathscr{T}$ be the functor which assigns to each object $A_{i}$ the classifying space $B E_{i}$. A morphism $f: A_{i} \rightarrow A_{j}$ is sent by $E_{\mathbf{A}}$ to the map $B E_{i} \rightarrow B E_{j}$ induced by the homomorphism $E_{i} \rightarrow E_{j}$ corresponding to $f$ under the unique basis-preserving isomorphisms $A_{i} \cong E_{i}$ and $A_{j} \cong E_{j}$.

6.2. Lemma. The map $\hat{\delta}_{i}: B E_{i} \rightarrow \widehat{B} \mathscr{C}\left(E_{i}\right), i=1, \ldots, 4,(\S 3)$ are the components [26, p. 215] of a dinatural transformation $\hat{\delta}: E_{\mathbf{A}} \stackrel{\ddot{\rightarrow}}{F}$; i.e., for each map $f: A_{i} \rightarrow A_{j}$ in $\mathbf{A}$ there is a commutative diagram

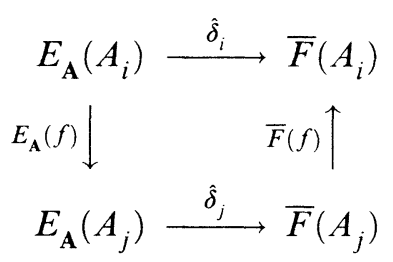

Proof. If $f$ is an automorphism of $A_{i}$ the above diagram commutes by $6.1(1)$, while if $f$ is one of the basic maps $f_{i}(2.1)$ it commutes by the definition of $\bar{F}\left(f_{i}\right)$. The lemma follows from the fact that these maps generate $\mathbf{A}$.

Now let $V$ be a rank four elementary abelian 2-group. Let $M_{\mathrm{A}}: \mathrm{A}^{\text {op }} \rightarrow$ $\operatorname{Ho}(\mathscr{T})$ be the functor which assigns to $A_{i}$ the function space $\operatorname{EMB}\left(B E_{i}, B V\right) \cong$ $\operatorname{Emb}\left(E_{i}, V\right) \times B V$. As in the discussion preceding 3.13 there are canonical maps $\kappa_{i, V}: M_{\mathbf{A}}\left(E_{i}\right) \rightarrow \widehat{B} \mathscr{C}\left(E_{i}\right)$.

6.3. Lemma. The maps $\kappa_{i, V}, i=1, \ldots, 4$, are the components of a natural transformation $\kappa_{V}: M_{\mathbf{A}} \rightarrow \bar{F}$.

Proof. We must show that for each morphism $f: A_{i} \rightarrow A_{j}$ in $\mathbf{A}$ and each $g \in \operatorname{EмB}\left(B E_{j}, B V\right)$ the following diagram in the homotopy category

$$
\begin{aligned}
& \operatorname{EMB}\left(B E_{i}, B V\right)_{g^{\prime}} \stackrel{\left(\kappa_{i, v^{\prime}}\right)_{g^{\prime}}}{\longrightarrow} \widehat{B} \mathscr{C}\left(E_{i}\right) \\
& (-) \cdot E_{\mathbf{A}} f \uparrow \quad \bar{F}(f) \uparrow \\
& \operatorname{EMB}\left(B E_{j}, B V\right)_{g} \underset{\left(\kappa_{j, V}\right)_{g}}{\longleftarrow} \widehat{B} \mathscr{C}\left(E_{j}\right)
\end{aligned}
$$

commutes, where $g^{\prime}=g \cdot E_{\mathbf{A}}(f)$ and subscripting a morphism denotes restriction to the indicated component of the domain. Each of the spaces on the left is equivalent by evaluation at the basepoint to $B V$, so the question is one of comparing two maps $B V \rightarrow \widehat{B} \mathscr{C}\left(E_{i}\right)$. The upper map $\left(\kappa_{i, V}\right)_{g^{\prime}}$ is the unique homotopy class which at the same time makes $H^{*} B V$ a finitely generated module over $H^{*} B \mathscr{C}\left(E_{i}\right)$ and satisfies $\left(\kappa_{i, V}\right)_{g^{\prime}} \cdot g^{\prime}=\hat{\delta}_{i}$. The other map, $\bar{F}(f) \cdot\left(\kappa_{j, V}\right)_{g}$, is the composite with $\bar{F}_{f}$ of the unique homotopy class 
which makes $H^{*} B V$ a finitely generated module over $H^{*} \widehat{B} \mathscr{C}\left(E_{j}\right)$ and satisfies $\left(\kappa_{j, V}\right)_{g} \cdot g=\hat{\delta}_{j}$. The two maps agree because $\bar{F}(f) \cdot \hat{\delta}_{j} \cdot E_{\mathbf{A}}(f)=\hat{\delta}_{i}$ (see 6.2) and $H^{*} \bar{F}(f)$ makes $H^{*} \widehat{B} \mathscr{C}\left(E_{i}\right)$ a finitely generated module over $H^{*} \widehat{B} \mathscr{C}\left(E_{j}\right)$. (This last follows from the fact that $\bar{F}(f)$ differs only by homotopy equivalences in the domain and range from a map induced by an inclusion $K_{1} \rightarrow K_{2}$ of compact Lie groups: any such map makes $H^{*} \widehat{B} K_{1}$ a finitely generated module over $H^{*} \widehat{B} K_{2}[29,2.4]$.)

In the same way as in 3.13 , the transformation $\kappa_{V}$ is equivariant with respect to the natural action of $\operatorname{Aut}(V)$ on $M_{\mathrm{A}}$ and the trivial action of $\operatorname{Aut}(V)$ on $\bar{F}$; it follows from 3.13 that the induced cohomology map $\kappa_{V}^{*}: H^{*} \bar{F} \rightarrow$ $\left(H^{*} M_{\mathrm{A}}\right)^{\mathrm{Aut}(V)}$ is an isomorphism. The equivalence $H^{*} \bar{F} \cong \alpha_{R}$ follows from the fact that $\left(H^{*} M_{\mathrm{A}}\right)^{\mathrm{Aut}(V)}$ is clearly up to equivalence the functor $\alpha_{R}$ of 2.3 .

\section{LIFTING TO THE CATEGORY OF SPACES}

In this section we will use the results of [6] to show that the functor $\bar{F}: \mathbf{A}^{\text {op }} \rightarrow$ $\operatorname{Ho}(\mathscr{T})$ can be lifted to a functor $F: \mathbf{A}^{\text {op }} \rightarrow \mathscr{T}$. This proves 2.5 and hence 1.1 .

Recall that a homotopy class of maps $f: X \rightarrow Y$ is said to be centric [6, $\S 1]$ if precomposition with $f$ induces a homotopy equivalence $\operatorname{AUT}(X)_{1} \rightarrow$ $\operatorname{MaP}(X, Y)_{f}$. A functor $\Phi: \mathbf{C} \rightarrow \operatorname{Ho}(\mathscr{T})$ is centric if $\Phi(f)$ is a centric map for each morphism $f$ of $\mathbf{C}$.

7.1. Proposition. The functor $\bar{F}: \mathbf{A}^{\text {op }} \rightarrow \operatorname{Ho}(\mathscr{T})$ is centric.

This is $[6,4.2]$. (Note that the partial completion functor which appears in the statement of $[6,4.2]$ agrees with the $\mathbf{F}_{2}$-completion functor in the cases at hand because the fundamental groups of the spaces involved are finite 2-groups [3, II, 5.2(iv)].)

\subsection{Lemma. The natural map (cf. $[6,4.5]$ )}

$$
B E_{i} \cong B \mathscr{Z}\left(\mathscr{C}\left(E_{i}\right)\right) \rightarrow \operatorname{AuT}\left(\widehat{B} \mathscr{C}\left(E_{i}\right)\right)_{1}
$$

is an equivalence for $i=2, \ldots, 4$.

Proof. The case $i=2$ is 5.5 . Cases $i=3$ and $i=4$ can be handled by explicit calculation (cf. $[6,5.3]$ ).

Let $\gamma_{j} \bar{F}(j \geq 1)$ be the functor $[6, \S 1]$ from $\mathbf{A}$ to the category of abelian groups which assigns to $A$ the group $\pi_{j} \operatorname{Aut}(\bar{F}(A))_{1}$; there is no basepoint problem with these homotopy groups because $\operatorname{AUT}(\bar{F}(A))_{1}$ is a simple space. For each map $f: A \rightarrow A^{\prime}$ in $\mathbf{A}$ the induced map $\gamma_{j} \bar{F}(f): \gamma_{j} \bar{F}(A) \rightarrow \gamma_{j} \bar{F}\left(A^{\prime}\right)$ is the composite

$$
\pi_{j} \operatorname{AUT}(\bar{F}(A))_{1} \stackrel{h_{\#}}{\rightarrow} \pi_{j} \operatorname{MAP}\left(\bar{F}\left(A^{\prime}\right), \bar{F}(A)\right)_{h} \stackrel{\left(h^{\#}\right)^{-1}}{\rightarrow} \pi_{j} \operatorname{AUT}\left(\bar{F}\left(A^{\prime}\right)\right)_{1}
$$

where $h$ is $\bar{F}(f), h_{\#}$ is induced by postcomposition with $h$, and $h^{\#}$ by precomposition. 
Recall from $\S 6$ that $E_{\mathbf{A}}$ is a functor which sends the object $A_{i}$ of $\mathbf{A}$ to $B E_{i}$. The natural action (7.2) of $B E_{i}$ on $\widehat{B} \mathscr{C}\left(E_{i}\right)$ gives rise to maps $\pi_{j} B E_{i} \rightarrow$ $\pi_{j} \operatorname{Aut}\left(\widehat{B} \mathscr{C}\left(E_{i}\right)\right)_{1}$.

7.3. Lemma. The above maps $\pi_{j} B E_{i} \rightarrow \pi_{j} \operatorname{AUT}\left(\widehat{B} \mathscr{C}\left(E_{i}\right)\right)_{1}$ are the components of a natural transformation $\pi_{j} E_{\mathbf{A}} \rightarrow \gamma_{j} \bar{F}$.

Proof. An examination of the definition of $\gamma_{k} \bar{F}$ shows that by 7.2 it is enough to check that for each morphism $f: A_{\alpha} \rightarrow A_{\beta}$ of $\mathbf{A}$ the following diagram in $\operatorname{Ho}(\mathscr{T})$ commutes:

$$
\begin{array}{lc}
B E_{\alpha} \times \widehat{B} \mathscr{C}\left(E_{\beta}\right) \stackrel{1 \times \bar{F}(f)}{\longrightarrow} B E_{\alpha} \times \widehat{B} \mathscr{C}\left(E_{\alpha}\right) \\
E_{\mathbf{A}}(f) \times 1 \mid \\
B E_{\beta} \times \widehat{B} \mathscr{C}\left(E_{\beta}\right) \stackrel{\bar{F}(f) \cdot m}{\longrightarrow} & \widehat{B} \mathscr{C}\left(E_{\alpha}\right)
\end{array}
$$

Here $m$ stands for the map $B E_{i} \times \widehat{B} \mathscr{C}\left(E_{i}\right) \rightarrow \widehat{B} \mathscr{C}\left(E_{i}\right)$ induced by the group homomorphism $E_{i} \times \mathscr{E}\left(E_{i}\right) \rightarrow \mathscr{C}\left(E_{i}\right)$. Restricting to the axes $B E_{\alpha} \times \mathrm{pt}$ and pt $\times \widehat{B} \mathscr{C}\left(E_{\beta}\right)$ respectively gives the diagrams

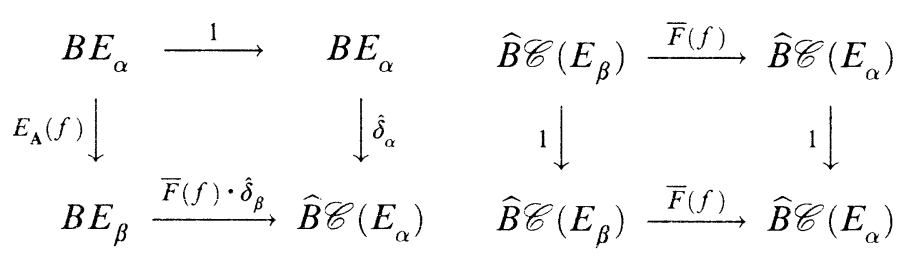

the first of which commutes by 6.2 and the second of which trivially commutes. The lemma follows from the fact that the basepoint evaluation map $\operatorname{MAP}\left(B E_{\alpha}, \widehat{B} \mathscr{C}\left(E_{\alpha}\right)\right)_{\hat{\delta}_{\alpha}} \rightarrow \widehat{B} \mathscr{C}\left(E_{\alpha}\right)$ is an equivalence ([14] and 5.3), so that a map $B E_{\alpha} \times \widehat{B} \mathscr{C}\left(E_{\beta}\right) \rightarrow \widehat{B} \mathscr{C}\left(E_{\alpha}\right)$ which agrees with $\hat{\delta}_{\alpha}$ on $B E_{\alpha} \times$ pt is actually determined up to homotopy by its restriction to $\mathrm{pt} \times \widehat{B} \mathscr{C}\left(E_{\beta}\right)$.

7.4. Lemma. The abelian groups $\lim ^{k} \pi_{j} E_{\mathbf{A}}$ vanish for $j, k>0$.

Proof. This is trivial if $j \neq 1$, since in that case the functor $\pi_{j} E_{\mathbf{A}}$ itself vanishes. Let $\Gamma=\operatorname{Aut}\left(A_{4}\right)$ and let $M$ be the $\Gamma$ module $A_{4}$ itself. Inspection then shows that the category $\mathbf{A}$ is equivalent to the category $\mathbf{A}_{\Gamma}$ of $\S 8$ in such a way that the functor $\pi_{1} E_{\mathrm{A}}$ corresponds to the functor $\alpha_{\Gamma, M}$ of 8.1 . Case $j=1$ of the lemma thus follows from 8.1.

7.5. Lemma. If $\gamma$ is a functor from $\mathbf{A}$ to the category of abelian groups such that $\gamma\left(A_{i}\right)=\{0\}$ for $i \neq 1$, then $\lim ^{k} \gamma$ vanishes for $k>0$.

Proof. This is an easy calculation with the standard cochain complex [3, XI, 6.2] for computing $\lim ^{*} \gamma$; in dimension $k$ this cochain complex contains a product

$$
\prod_{a_{0} \rightarrow a_{1} \rightarrow \cdots \rightarrow a_{k}} \gamma\left(a_{k}\right)
$$


indexed by $k$-simplices of the nerve of $\mathbf{A}$. Under the given circumstances this is the complex for computing the singular cohomology of a point with coefficients in $\gamma\left(A_{0}\right)$.

7.6. Lemma. The abelian groups $\lim ^{k} \gamma_{j} \bar{F}$ vanish for $j, k>0$.

Proof. By 7.2 the natural transformation of 7.3 is an isomorphism at the objects $A_{i}, i \neq 1$, of $\mathbf{A}$. It is easy to check that the natural transformation is a monomorphism at $A_{1}$; for $j>1$ this is the case because the domain group is zero, while for $j=1$ it follows from naturality and the fact that for any morphism $A_{1} \rightarrow A_{2}$ of $\mathbf{A}$ the induced map $E_{1} \rightarrow E_{2}$ is injective. If $\gamma$ is the cokernel of this natural transformation, then by 7.5 the groups $\lim ^{k} \gamma$ vanish for $k>0$. The lemma now follows from 7.4 and the long exact $\longleftarrow^{*}$ sequence $[3, \mathrm{XI}, 6.1]$ associated to a short exact sequence of functors.

Remark. Jackowski, McClure, and Oliver have recently shown that 7.2 also holds for $i=1$; given this result, which is difficult, 7.6 follows directly from 7.4.

7.7. Proposition. The functor $\bar{F}: \mathbf{A}^{\text {op }} \rightarrow \mathrm{Ho}(\mathscr{T})$ can be lifted to a functor $F: \mathbf{A}^{\text {op }} \rightarrow \mathscr{T}$.

Proof. Given 7.6, this is a consequence of the main theorem of [6]. In fact, the results of [6] show that up to a strong notion of equivalence there is only one lift $F$ of $\bar{F}$.

Remark. Given a centric diagram $\bar{F}$ in the homotopy category such that the realization obstruction groups [6] for $\bar{F}$ vanish (7.6) and such that $H^{*} \bar{F}$ is equivalent to $\alpha_{R}(\S 2)$, Oliver has shown how to construct $B \mathrm{DI}(4)$ directly; this provides a proof of 1.1 which does not use 7.7 .

\section{DERIVED FUNCTORS OF THE INVERSE LIMIT FUNCTOR}

In this section we will prove a certain vanishing theorem for the higher derived functors $\lim ^{i}[3, \mathrm{XI}, \S 6]$ of the inverse limit functor. This vanishing theorem figures in the proof of 2.4 and in the proof of 7.4. We work in greater generality than we strictly need in order to emphasize the analogy with [13].

Let $p$ be a prime number (not necessarily 2), $V$ a finite-dimensional vector space over $\mathbf{F}_{p}, \Gamma \subset \operatorname{Aut}(V)$ a subgroup and $M$ a module over $\mathbf{F}_{p}[\Gamma]$. For any $\mathbf{F}_{p}$ vector space $A$ the group $\Gamma$ acts by composition on $\operatorname{Hom}(A, V)$. Define $\mathbf{A}_{\Gamma}$ to be the category of pairs $(A, O)$ where $A$ is a nonzero $\mathbf{F}_{p}$ vector space and $O$ is a $\Gamma$ orbit of monomorphisms $A \rightarrow V$; a map $(A, O) \rightarrow\left(A^{\prime}, O^{\prime}\right)$ is a monomorphism $f: A \rightarrow A^{\prime}$ such that $g \circ f \in O$ whenever $g \in O^{\prime}$.

Let $\alpha_{\Gamma, M}$ be the functor from $\mathbf{A}_{\Gamma}$ to abelian groups which assigns to $(A, O)$ the vector space of $\Gamma$-maps $O \rightarrow M$. For any object $x$ of $\mathbf{A}_{\Gamma}$ there is an evident map $M^{\Gamma} \rightarrow \alpha_{\Gamma, M}(x)$; taken together these induce a map $M^{\Gamma} \rightarrow \lim _{\longleftarrow} \alpha_{\Gamma, M}$.

8.1. Proposition. The above map $M^{\Gamma} \rightarrow \underset{\lim }{\longleftarrow} \alpha_{\Gamma, M}$ is an isomorphism. The groups $\lim ^{i} \alpha_{\Gamma, M}$ vanish for $i \geq 1$. 
Remark. Let $\alpha_{\Gamma, M}^{k}$ be the functor from $\mathbf{A}_{\Gamma}$ to abelian groups which assigns to $(A, O)$ the group $\operatorname{Ext}_{\mathbf{F}_{p}[\Gamma]}^{k}\left(\mathbf{F}_{p}[O], M\right)$. (Note that $\alpha_{\Gamma, M}^{0}=\alpha_{\Gamma, M}$ and that $\alpha_{\Gamma, M}^{k}(A, O)$ is isomorphic to $H^{k}\left(\Gamma_{f}, M\right)$, where $\Gamma_{f} \subset \Gamma$ is the isotropy subgroup of any $f \in O$.) The proof of 8.1 shows that $\lim _{\Gamma, M}^{k} \cong \alpha^{k}(\Gamma, M)$ and that $\stackrel{\lim }{ }^{i} \alpha_{\Gamma, M}^{k}=0$ for $i \geq 1$.

8.2. Lemma. The conclusion of 8.1 is true under the additional assumption that $\Gamma$ is a p-group.

Proof (cf. [13, proof of 4.10]). Since $\Gamma$ is a finite $p$-group, $V^{\Gamma} \neq\{0\}$. Denote by $F$ the object of $\mathbf{A}_{\Gamma}$ corresponding to the inclusion $l: V^{\Gamma} \rightarrow V$.

Let $F \downarrow \mathbf{A}_{\Gamma}$ be the under category [26, p. 46] and $j: F \downarrow \mathbf{A}_{\Gamma} \rightarrow \mathbf{A}_{\Gamma}$ the forgetful functor. The category $F \downarrow \mathbf{A}_{\Gamma}$ has the identity map of $F$ as an initial object and it is clear that the natural map

$$
M^{\Gamma} \rightarrow \alpha_{\Gamma, M}(F)=\alpha_{\Gamma, M}(j(F \stackrel{1}{\rightarrow} F))
$$

is an isomorphism; it follows [3, XI, 7.2 and 9.2] that the natural map $M^{\Gamma} \rightarrow$ $\lim \left(\alpha_{\Gamma, M} \cdot j\right)$ is an isomorphism and that $\lim ^{i}\left(\alpha_{\Gamma, M} \cdot j\right)$ vanishes for $i \geq 1$. Given an arbitrary object $x=(A, O)$ of $\overleftarrow{\mathbf{A}_{\Gamma}}$ choose $f \in O$ and let $\sigma(x)$ be the pair $\left(A^{\prime}, O^{\prime}\right)$ where $A^{\prime}$ is the quotient of $A \oplus V^{\Gamma}$ by the kernel of the sum map $f+l: A \oplus V^{\Gamma} \rightarrow V$ and $O^{\prime}$ is the orbit of the monomorphism $A^{\prime} \rightarrow V$. It is easy to check that $\sigma(x)$ does not depend on the choice of orbit element $f$, that $x \mapsto \sigma(x)$ produces a functor $\sigma: \mathbf{A}_{\Gamma} \rightarrow F \downarrow \mathbf{A}_{\Gamma}$, and that the map $A \rightarrow A \oplus V^{\Gamma} \rightarrow A^{\prime}$ induces a natural transformation $\tau$ from the identity functor of $\mathbf{A}_{\Gamma}$ to the composite $j \cdot \sigma$. By inspection the natural transformation from $\alpha_{\Gamma, M}$ to $\alpha_{\Gamma, M} \cdot j \cdot \sigma$ induced by $\tau$ is a natural equivalence (cf. 8.1).

Now let $x$ be an object of $F \downarrow \mathbf{A}_{\Gamma}, y$ an object of $\mathbf{A}_{\Gamma}$ and $\sigma(y) \rightarrow x$ a map of $F \downarrow \mathbf{A}_{\Gamma}$. It is clear that there is a unique map $w: y \rightarrow j(x)$ in $\mathbf{A}_{\Gamma}$ such that the given map $\sigma(y) \rightarrow x$ is the composite of $\sigma(w)$ with the evident natural isomorphism $\sigma(j(x)) \cong x$ (in fact, $\sigma$ is left adjoint to $j$ ). This shows that the over category $\sigma \downarrow x$ has a terminal object and therefore a contractible nerve [3, $\mathrm{XI}, \S 2]$; consequently, the functor $\sigma$ is left cofinal [3, XI, §9]. The proposition now follows directly from the fact [3, XI, 7.2 and 9.2] that the natural maps $\lim ^{i}\left(\alpha_{\Gamma, M} \cdot j\right) \rightarrow \lim ^{i}\left(\alpha_{\Gamma, M} \cdot j \cdot \sigma\right) \cong \lim ^{i} \alpha_{\Gamma, M}$ are isomorphisms for all $i$. (The isomorphism $\lim ^{i}\left(\alpha_{\Gamma, M} \cdot j\right) \cong \lim ^{i} \alpha_{\Gamma, M}$ is in fact a general consequence of the fact that $j$ has a left adjoint [17].)

Let $\mathbf{B}$ be the category whose objects are nontrivial vector spaces of dimension $\leq \operatorname{dim}(V)$ and whose morphisms are vector space monomorphisms. Let $\beta_{\Gamma, M}$ be the functor from $B$ to abelian groups which assigns to each object $A$ the vector space of $\Gamma$-maps $\operatorname{Emb}(A, V) \rightarrow M$ (here as in $\S 5 \operatorname{Emb}(A, V)$ is the set of monomorphisms $A \rightarrow V)$. The unique $\Gamma$-map $\operatorname{Emb}(A, V) \rightarrow *$ induces for each $A$ a map $M^{\Gamma} \rightarrow \beta_{\Gamma, M}(A)$; these combine to give a map $M^{\Gamma} \rightarrow \underset{\lim }{\longleftarrow} \beta_{\Gamma, M}$. 
8.3. Lemma. These are natural isomorphisms $\lim ^{i} \alpha_{\Gamma, M} \rightarrow \lim ^{i} \beta_{\Gamma, M}$ for all $i \geq 0$. Under the isomorphisms the natural map $M^{\Gamma} \rightarrow \lim \alpha_{\Gamma, M}$ corresponds to the natural map $M^{\Gamma} \rightarrow \underset{\lim }{\longleftarrow} \beta_{\Gamma, M}$.

The proof of this is identical to the proof of $[13,2.3]$. The standard cochain complexes which compute the two higher limits involved are isomorphic.

Proof of 8.1 (cf. [13, proof of 1.2]). By 8.3 it is enough to prove that the natural map $M^{\Gamma} \rightarrow \lim _{\Gamma, M}$ is an isomorphism and that $\lim ^{i} \beta_{\Gamma, M}$ vanishes for $i \geq 1$. Let $\Pi \subset \Gamma$ be a $p$-Sylow subgroup. Since the index of $\Pi$ in $\Gamma$ is prime to $p$, the transfer construction $\left[4\right.$, p. 80] exhibits $\beta_{\Gamma, M}$ as a natural retract of $\beta_{\Pi, M}$. This implies that $\lim ^{i} \beta_{\Gamma, M}$ is a retract of $\lim ^{i} \beta_{\Pi, M}(i \geq 0)$. The desired result now follows from 8.2, since a retract of an isomorphism is an isomorphism $(i=0)$ and a retract of $\{0\}$ is $\{0\} \quad(i \geq 1)$.

\section{ACKNOWLEDGMENT}

The authors would like to thank D. Benson, R. Oliver, R. Kane, and the referee for very helpful comments.

\section{REFERENCES}

1. M. F. Atiyah and I. G. Macdonald, Introduction to commutative algebra, Addison-Wesley, New York, 1969.

2. A. Borel, Sur l'homologie et la cohomologie des groupes de Lie compacts connexes, Amer. J. Math. 76 (1954), 273-342.

3. A. K. Bousfield and D. M. Kan, Homotopy limits, completions and localizations, Lecture Notes in Math., vol. 304, Springer-Verlag, Berlin-Heidelberg-New York, 1972.

4. K. S. Brown, Cohomology of groups, Graduate Texts in Math., vol. 87, Springer-Verlag, Berlin-Heidelberg-New York, 1982.

5. A. Clark and J. Ewing, The realization of polynomial algebras as cohomology rings, Pacific J. Math. 50 (1974), 425-434.

6. W. G. Dwyer and D. M. Kan, Centric maps and realization of diagrams in the homotopy category, Preprint, Univ. of Notre Dame, 1989.

7. W. G. Dwyer, D. M. Kan, and J. H. Smith, Towers of fibrations and homotopical wreath products, J. Pure Appl. Algebra 56 (1989), 9-28.

8. W. G. Dwyer, H. R. Miller, and C. W. Wilkerson, The homotopic uniqueness of $B S^{3}$, Algebraic Topology, Barcelona 1986, Lecture Notes in Math., vol. 1298, Springer-Verlag, Berlin-Heidelberg-New York, 1987, pp. 90-105.

9. __ Homotopical uniqueness of classifying spaces, Topology 31 (1992), 29-45.

10. W. G. Dwyer and G. Mislin, On the homotopy type of the components of $\operatorname{Map}_{*}\left(B S^{3}, B S^{3}\right)$, Algebraic Topology, Barcelona 1986, Lectures Notes in Math., vol. 1298, Springer-Verlag, Berlin-Heidelberg-New York, 1987, pp. 82-89.

11. W. G. Dwyer and C. W. Wilkerson, Smith theory and the functor T, Comment. Math. Helv. 66 (1991), 1-17.

12. Spaces of null homotopic maps (Proc. of the 1988 Luminy Conference), Asterisque, no. 191, Soc. Math. France, Paris, 1990, pp. 97-108.

13. _ A cohomology decomposition theorem, Topology 31 (1992), 433-443. 
14. W. G. Dwyer and A. Zabrodsky, Maps between classifying spaces, Algebraic Topology, Barcelona 1986, Lecture Notes in Math., vol. 1298, Springer-Verlag, Berlin-HeidelbergNew York, 1987, pp. 106-119.

15. P. Gabriel and M. Zisman, Calculus of fractions and homotopy theory, Ergeb. Math. Grenzgeb. (3), vol. 35, Springer-Verlag, Berlin-Heidelberg-New York, 1967.

16. N. Iwase, On the splitting of mapping spaces between classifying spaces. I, Publ. Res. Inst. Math. Sci. 26 (1987), 445-453.

17. S. Jackowski and J. E. McClure, A homotopy decomposition theorem for classifying spaces of compact Lie groups, Topology 31 (1992), 113-132.

18. A. Jeanneret and U. Suter, Réalisation topologique de certaines algèbres associées aux algèbres de Dickson, Preprint.

19. J. Lannes, Sur la cohomologie modulo $p$ des p-groupes Abéliens élémentaires, Homotopy Theory, Proc. Durham Sympos. 1985 (E. Rees and J. D. S. Jones, eds.), Cambridge Univ. Press, Cambridge, 1987.

20. _ Cohomology of groups and function spaces, Preprint, École Polytechnique, 1987.

21. Sur les espaces fonctionnels dont la source est le classifiant d'un p-groupe abélien élémentaire, Preprint, École Polytechnique, 1990.

22. J. Lannes and L. Schwartz, Sur la structure des $\mathscr{A}$-modules instables injectifs, Topology 28 (1989), 153-169.

23. J. P. Lin, Correction to the paper "Primitivity of the $c_{2}$ invariant", J. Pure Appl. Algebra (to appear).

24. J. P. Lin and F. Williams, On 14-connected finite H-spaces, Israel J. Math. 66 (1989), 274-288.

25. __ On 6-connected finite H-spaces with 2-torsion, Topology 28 (1989), 7-34.

26. S. Mac Lane, Categories for the working mathematician, Graduate Texts in Math., vol. 5, Springer-Verlag, Berlin-Heidelberg-New York, 1971.

27. D. Notbohm, Abbildungen zwischen klassifizierenden Räume, Dissertation, Göttingen, 1988.

28. D. G. Quillen, The mod 2 cohomology rings of extra-special 2-groups and the spinor groups, Math. Ann. 194 (1971), 197-212.

29. __ The spectrum of an equivariant cohomology ring. I, Ann. of Math. (2) 94 (1971), 549-572.

30. J. P. Serre, Sur les sous-groupes abéliens des groupes de Lie compacts, Exposé no. 24 (7.6.55), Séminaire Sophus LIE, École Norm. Sup., 1954/55.

31. L. Smith and R. M. Switzer, Realizability and non-realizability of the Dickson algebras as cohomology rings, Proc. Amer. Math. Soc. 89 (1983), 303-313.

32. C. W. Wilkerson, A primer on the Dickson invariants, Contemp. Math., vol. 19, Amer. Math. Soc., Providence, RI, 1983, pp. 233-246.

Department of Mathematics, University of Notre Dame, Notre Dame, Indiana 46556 E-mail address: William.G.Dwyer.1@nd.edu

Department of Mathematics, Purdue University, West Lafayette, Indiana 47907

E-mail address: wilker@math.purdue.edu 